INTER NATIONAL MONETARY FUND

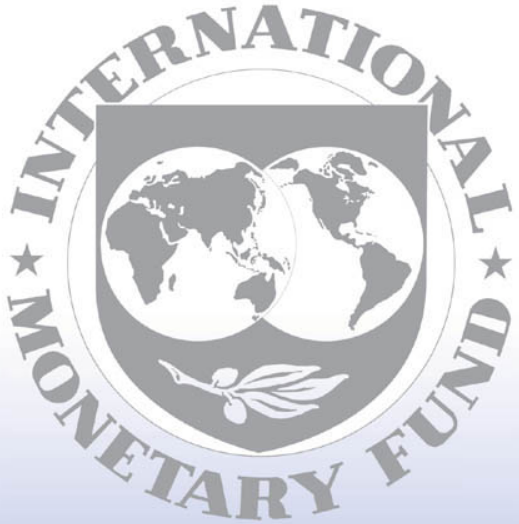

Staff

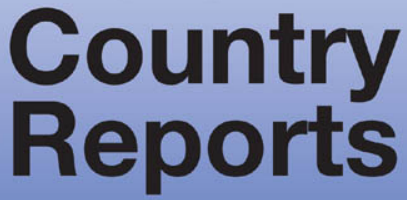




\section{The Kingdom of Swaziland: Statistical Appendix}

This Statistical Appendix paper for The Kingdom of Swaziland was prepared by a staff team of the International Monetary Fund as background documentation for the periodic consultation with the member country. It is based on the information available at the time it was completed on January 24, 2006. The views expressed in this document are those of the staff team and do not necessarily reflect the views of the government of The Kingdom of Swaziland or the Executive Board of the IMF.

The policy of publication of staff reports and other documents by the IMF allows for the deletion of market-sensitive information.

To assist the IMF in evaluating the publication policy, reader comments are invited and may be sent by e-mail to publicationpolicy@imf.org.

Copies of this report are available to the public from

International Monetary Fund • Publication Services

700 19th Street, N.W. • Washington, D.C. 20431

Telephone: (202) 6237430 • Telefax: (202) 6237201

E-mail: publications@imf.org • Internet: http://www.imf.org

Price: $\$ 15.00$ a copy

\section{International Monetary Fund Washington, D.C.}


This page intentionally left blank

CInternational Monetary Fund. Not for Redistribution 


\section{INTERNATIONAL MONETARY FUND}

\section{THE KINGDOM OF SWAZILAND}

\section{Statistical Appendix}

Prepared by the African Department

Prepared by a staff team consisting of

Messrs. Wang (Head), Gons, and Haacker, and Ms. Masha (all AFR)

Approved by the African Department

January 24, 2006

Contents

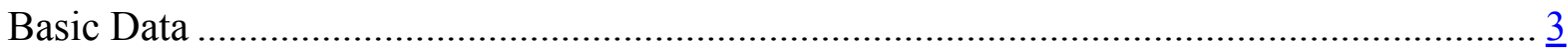

Swaziland's External Competitiveness in the Context of the Common Monetary Area ........... 4

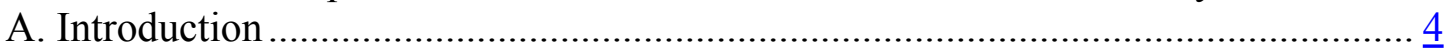

B. Objectives and Key Features of the CMA............................................................ 4

C. Indicators of External Competitiveness ……………......................................... $\underline{6}$

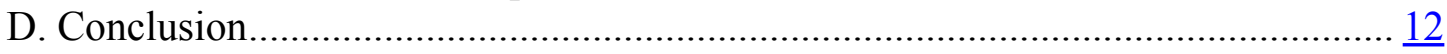

Text Tables

I.1. Corruption Perception Index.................................................................................. 11

Statistical Appendix Tables

1. Gross Domestic Product by Sector of Origin at 2000 Prices, 2000-2004 ....................... 17

2. Gross Domestic Product by Sector of Origin at Current Prices, 2000-2004 …................ 18

3. Gross Domestic Product by Expenditure Category at Current Prices, 2000-2004 .......... $\frac{19}{20}$

4. Population and Labor Force Estimates, 2000-2003 …............................................... 20

5. Summary of Developments in Crop Production, 2000/01-2004/05 .............................. 21

6. Developments in Sugar Production, Processing, and Consumption, 2000/01-2004/05 .. 22

7. Mineral Production and Exports, 2000-2004 ……................................................. 23

8. Index of Industrial Production, 2001-2003 ........................................................... 24

9. Electrical Power Generation and Demand, 2000-2004 .............................................. 25

10. Retail Price Indices of Urban Families, 2001-2005 ………….................................. $\frac{26}{26}$

11. Minimum Wages for General Workers in Selected Industries, 2001-2003 .................... $\frac{27}{27}$

12. Paid Employment in the Private and Public Sectors by Industry, 2000-2003 ................. 28

13. Summary of Central Government Operations, 2000/01-2004/05 ………………........... 29

14. Central Government Revenue and Grants, 2000/01-2004/05 ........................................ $\underline{30}$

15. Functional Classification of Central Government Expenditure and Net Lending, 2000/01-2004/05 
16. Economic Classification of Government Current Expenditure, 2000/01-2004/05 …....... $\underline{32}$

17. Capital Expenditure by Sector, 2000/01-2004/05 …….............................................. $\frac{33}{34}$

18. Government Transfer Payments, 2000/01-2003/04 ……………………………….... 34

19. Flow of Funds From the Government to Public Enterprises 2000/01-2004/05 ……........ $\underline{35}$

20. Outstanding Domestic Government Debt by Type of Instrument, 2000-2004 ................. $\underline{36}$

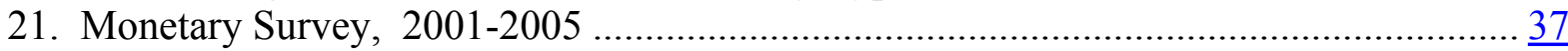

22. Assets and Liabilities of the Central Bank of Swaziland, 2000 - Nov. 2005.................... $\underline{38}$

23. Assets and Liabilities of Commercial Banks, 2000 - Nov. 2005 .................................... $\underline{39}$

24. Commercial Banks' Deposits by Sector, Dec. 2001 - Sept. 2005 .................................... $\frac{40}{41}$

25. Commercial Banks' Liquidity Position, Dec. 2001 - Sept. 2005 .................................... 41

26. Commercial Bank Loans and Advances by Category of Borrower, Dec. 2000 -

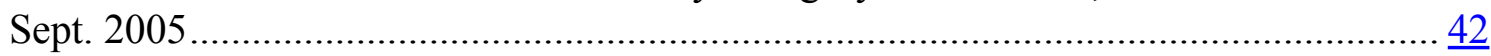

27. Interest Rates in Swaziland and South Africa, Dec. 2001 - Nov. 2005 .......................... 43

28. Balance of Payments, 2000-2004 ............................................................................

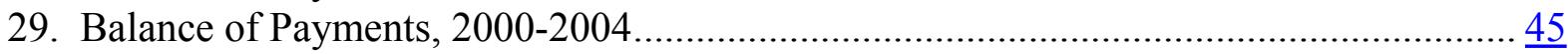

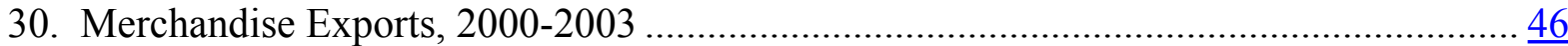

31. Sugar Exports by Volume, Value, and Unit Price, 1999-2004 ……............................ 47

32. Other Principal Exports by Volume, Value, and Unit Price, 2000-2004 ........................ 48

33. Merchandise Imports, 2001-2003 …………….................................................. 49

34. Services and Income Account, 2001-2004 .............................................................

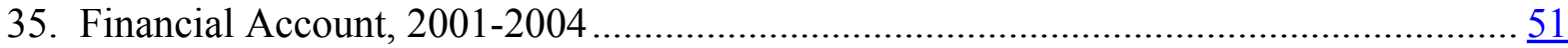

36. Public Sector External Debt, 2000/01-2004/05 …......................................................... $\frac{52}{53}$

37. Public Sector External Debt Service, 2000/01-2004/05 ……………………................. 53

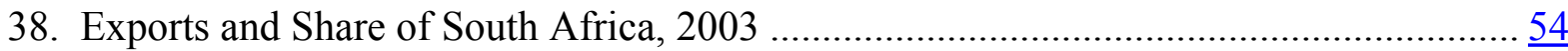

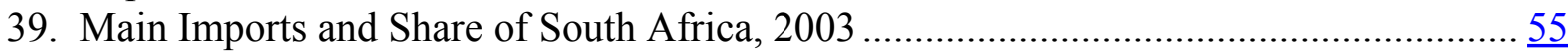

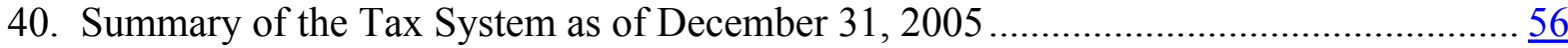

Figures

I.1. Real and Nominal Effective Exchange Rates, Jan 2001-Sep 2005 ……........................ $\underline{8}$

I.2. Montly Minimum Wage in Manufacturing and Processing ........................................... $\frac{9}{9}$

I.3. Garment Sector Wages for Selected Countries, 2002 ................................................... 9

I.4. Sugarcane and Maize Yield .................................................................................. 


\section{BASIC Data}

\section{Swaziland: Basic Data}

Area

Population (2005)

Population growth rate (2005)

Formal employment (2003)

IMF Position (November 30, 2005)

Quota

Fund holdings of emalangeni

Holdings of SDRs

Exchange rate
17,364 square kilometers

$1,045,000$

0 percent

102,000

SDR 50.70 million

SDR 44.15 million

SDR 2.48 million

$\mathrm{US} \$ 1=\mathrm{E} 6.32$

\begin{tabular}{|c|c|c|c|c|c|}
\hline & 2000 & 2001 & 2002 & 2003 & $\begin{array}{c}2004 \\
\text { Prel. }\end{array}$ \\
\hline & \multicolumn{5}{|c|}{ (Annual percentage change, unless stated otherwise) } \\
\hline \multicolumn{6}{|l|}{ National Income and Prices } \\
\hline GDP at constant prices & 2.6 & 1.6 & 2.9 & 2.4 & 2.1 \\
\hline GDP per capita at constant prices & 0.0 & -1.0 & 0.3 & 0.1 & -0.1 \\
\hline GDP deflator & 11.6 & 10.8 & 12.6 & 12.1 & 3.4 \\
\hline CPI (period average) & 7.2 & 7.5 & 11.7 & 7.4 & 3.4 \\
\hline CPI (end of period) & 6.4 & 10.8 & 11.5 & 4.6 & 3.2 \\
\hline Unemployment rate (in percent) & 31.3 & 31.3 & 30.0 & 29.0 & 30.0 \\
\hline \multicolumn{6}{|l|}{ External sector } \\
\hline Current account balance (in millions of U.S. dollars) & -75.0 & -56.8 & 57.6 & 36.3 & 40.0 \\
\hline Trade balance (in millions of U.S. dollars) & -31.2 & -22.5 & -0.3 & -0.9 & -13.1 \\
\hline Exports, f.o.b. 1/ & 3.0 & 48.3 & 16.0 & 3.3 & 2.8 \\
\hline Imports, f.o.b. 1/ & -6.9 & 33.7 & -9.2 & -9.8 & -2.4 \\
\hline Nominal effective exchange rate $2 /$ & -1.9 & -5.1 & 0.1 & 4.4 & 1.3 \\
\hline Real effective exchange rate $2 /$ & -1.9 & 1.7 & -0.5 & 11.8 & 1.2 \\
\hline \multicolumn{6}{|l|}{ Money and credit } \\
\hline Broad money & -6.6 & 10.7 & 13.1 & 14.1 & 10.4 \\
\hline Prime lending rate (in percent; end of period) & 14.0 & 12.5 & 16.5 & 11.5 & 11.0 \\
\hline Interest rates on 12-month time deposits (in percent; end of period) & 7.0 & 6.3 & 9.5 & 4.2 & 4.1 \\
\hline & \multicolumn{5}{|c|}{ (In percent of GDP) } \\
\hline Gross national savings & 13.2 & 13.9 & 24.6 & 19.9 & 19.0 \\
\hline Of which: government & 4.8 & 5.2 & 3.0 & 3.3 & 3.4 \\
\hline Gross domestic investment & 18.6 & 18.4 & 19.8 & 18.0 & 17.4 \\
\hline Of which: government & 6.3 & 8.0 & 7.4 & 5.7 & 7.7 \\
\hline \multicolumn{6}{|l|}{ Central government finance (fiscal year) 3/ } \\
\hline Total revenue and grants & 28.4 & 27.6 & 26.3 & 26.3 & 29.3 \\
\hline Of which: South African Customs Union (SACU) receipts & 14.2 & 13.3 & 12.4 & 12.6 & 16.8 \\
\hline Total expenditure and net lending & 29.9 & 30.6 & 30.8 & 29.0 & 33.6 \\
\hline Current expenditure and net lending & 23.8 & 22.6 & 23.4 & 23.1 & 26.0 \\
\hline Central government balance (including grants) & -1.4 & -3.1 & -4.5 & -2.7 & -4.3 \\
\hline Primary balance (including grants) & -0.8 & -2.1 & -3.3 & -1.6 & -3.3 \\
\hline \multicolumn{6}{|l|}{ External sector } \\
\hline Current account balance & -5.4 & -4.5 & 4.8 & 1.9 & 1.6 \\
\hline Trade balance (merchandise goods) & -9.8 & -6.5 & 7.6 & 2.2 & -1.3 \\
\hline Capital and financial account balance & 1.2 & -9.9 & -6.6 & -3.7 & 0.4 \\
\hline Overall balance 4/ & -0.5 & -4.5 & -2.2 & -2.3 & -0.7 \\
\hline External debt & 25.8 & 22.9 & 29.4 & 21.7 & 22.6 \\
\hline \multicolumn{6}{|l|}{ Memorandum items: } \\
\hline GDP in current prices (in millions of emalangeni) ${ }^{5 /}$ & 9,638 & 10,846 & 12,560 & 14,422 & 16,262 \\
\hline Overall balance of payments (in millions of U.S. dollars) & -6 & -56 & -25 & -21 & 45 \\
\hline Net official international reserves (in millions of U.S. dollars) & 293 & 228 & 217 & 212 & 258 \\
\hline (in months of imports of goods and nonfactor services) & 2.6 & 2.0 & 2.3 & 1.5 & 1.3 \\
\hline Total external debt (in millions of U.S. dollars) & 358 & 288 & 351 & 413 & 571 \\
\hline
\end{tabular}

Sources: Swazi authorities.

1/ In U.S. dollars.

2/ IMF Information Notice System trade-weighted; end of period.

3/ The fiscal year runs from April 1 to March 31.

4/ Excluding net errors and omissions.

5/ Under review by the CSO; data on indirect taxes used for estimation of GDP may contain errors and are subject to downward revisions based on the review.

\section{CInternational Monetary Fund. Not for Redistribution}




\section{SWAZILAND'S EXTERNAL COMPETITIVENESS IN THE CONTEXT OF THE COMMON Monetary AReA ${ }^{1}$}

\section{A. Introduction}

1. Swaziland is a small, open, land-locked economy within the Common Monetary Area (CMA) and the South African Customs Union (SACU). ${ }^{2}$ There are no exchange controls among CMA countries, while trade among SACU countries is free of tariffs and quotas. The SACU joint customs and excise pool provides about half of Swaziland's fiscal revenues. Swaziland's main exports are sugar and sugar-based products, wood pulp and garments. South Africa absorbs about 60 percent of Swazi exports, and provides about 80 percent of the country's imports. Although Swaziland is a low middle-income country, poverty is widespread, aggravated by food shortages in some parts of the country and a severe HIV/AIDS epidemic.

2. Except for 2002, when the garment sector grew rapidly, economic growth has decelerated since the mid-1990s while the external current account has deteriorated. At the same time, foreign direct investment (FDI) declined from an annual average of 7.1 percent of GDP in 1985-93 to 4.7 percent of GDP in 1994-2002 and, after turning negative in 2003, amounted to 2.5 percent of GDP in 2004. External factors, such as South Africa's democratization since 1994, as well as domestic factors, e.g., governance issues, have contributed to the slowdown. Taken together, these developments suggest a weakening of Swaziland's external competitiveness. After describing some key features of the CMA and their policy implications for Swaziland (Section B), this chapter, to the extent that data availability allows, discusses (in Section C) some aspects of Swaziland's external competitiveness focusing on (i) trade preferences; (ii) exchange rate developments; (iii) wage costs and productivity; and (iv) nonwage costs and the investment climate. Section D concludes the chapter.

\section{B. Objectives and Key Features of the $\mathrm{CMA}^{3}$}

3. The CMA was established in April 1986, succeeding the Rand Monetary Area (RMA) that was created in December 1974. The CMA's objectives are to confer the advantages of a common monetary area on its members, provide for the sustained economic development of the CMA as a whole, encourage the advancement of the less-developed members, and afford to all parties equitable benefits arising from the maintenance and development of the CMA.

\footnotetext{
${ }^{1}$ Prepared by Jacob Gons.

${ }^{2}$ CMA members include Lesotho, Namibia (since 1990), South Africa, and Swaziland. SACU members include the CMA countries plus Botswana.

${ }^{3}$ A more complete description of CMA's features is contained in the Appendix.
} 
4. Article 2 of the CMA agreement gives each of the three small member countries (Lesotho, Namibia, and Swaziland) the right to issue national currencies. The local currencies (in Swaziland, the lilangeni) issued by the three members are legal tender in their respective countries only. The South African rand, however, is legal tender throughout the CMA. ${ }^{4}$ Like the currencies of the other small CMA members, the lilangeni is pegged to the rand at par.

5. Under the terms of the CMA Agreement (Article 3), no restrictions can be imposed on the transfer of funds, whether for current or capital transactions, to or from any member country.

6. Article 5 of the CMA Agreement requires the smaller countries' exchange control regulations to be similar, in all material aspects, to those in effect in South Africa.

\section{Policy Implications of Swaziland's Membership in the CMA}

7. The peg of the lilangeni to the rand, the parallel circulation of the lilangeni and the rand, currency convertibility, and regional capital mobility imply that Swaziland (like the other small CMA countries) does not have independent control of its money supply. The demand for emalangeni depends in large part on the public's confidence in the exchange rate parity given the extensive financial linkages between Swaziland and South Africa. ${ }^{5}$ Swaziland's monetary base basically expands (or contracts) in line with central bank purchases (or sales) of foreign exchange. Given the small size of its economy relative to that of South Africa and their extensive financial links, interest rate movements in Swaziland largely mirror those in South Africa.

8. Swaziland's exchange rate arrangement under the CMA has certain characteristics of a currency board, with the Central Bank of Swaziland (CBS) converting at the fixed rate emalangeni into rand and vice versa. However, a currency board is typically prohibited by law from acquiring domestic assets, so all the currency it issues is automatically and fully backed by foreign reserves. ${ }^{6}$ There is no such legal restriction for Swaziland under the CMA. Thus, following draw downs of government deposits in recent years to help finance fiscal deficits, the CBS has been recording an increase in its net domestic assets and, given the

\footnotetext{
${ }^{4}$ In Swaziland, the rand was reinstated as legal tender in 2003, after a 17-year interruption.

${ }^{5}$ These linkages are facilitated by the fact that from the four banks that comprise Swaziland's banking system, three (holding some 80 percent of banking system assets) are South African. These banks' liquidity is managed through their respective head offices.

${ }^{6}$ See Humpage and McIntire (1995).
} 
demand for emalangeni, diminishing levels of its net international reserves. ${ }^{7}$ As of endSeptember 2005, Swaziland's international reserves covered only 1.1 months of imports.

9. Given the exchange rate peg and lack of monetary independence, Swaziland will have to rely on fiscal and structural policy measures to achieve its economic goals and preserve, or improve, its external competitiveness. The next section discusses some indicators of Swaziland's external competitiveness.

\section{Indicators of External Competitiveness}

\section{Trade preferences}

10. The existence of several preferential trade arrangements represents a key factor in Swaziland's external competitiveness. ${ }^{8}$ The Swazi economy benefits from preferential access to the European Union (EU) under the Cotonou agreement and, since 2000, to the United States (U.S.) under the African Growth and Opportunity Act (AGOA). However, both the EU and the U.S. have maintained quotas on sugar imports.

11. Sugar accounts for some 12 percent of Swaziland's exports, about half of which is exported to other SACU countries. Sugar is exported to the EU under two preferential agreements: the Sugar Protocol (100,000-130,000 tons per year) and the Special Preference Sugar (SPS) (about 40,000 tons per year), at prices that are more than three times higher than those in unprotected sugar markets. Exports to the EU amount to about one quarter of total sugar exports (including to SACU countries) in volume terms, and 40 percent by value.

12. Swaziland historically has had a small garment sector that mainly catered to the South African market. The coming into force of AGOA's apparel provisions in 2001 explains its recent rise, through foreign direct investment notably from the Far East. At its peak, in early 2004, the sector provided about one quarter of formal employment. The bulk of the garment sector's output consists of quota-constrained basic items (e.g., trousers and T-shirts), sold to high-volume discount chains in the U.S.. Swaziland's garment exports have particularly benefited from AGOA's "third party fabric provision" granted to least-developed countries (LDC's). Under this provision, Swaziland is allowed to import inputs from nonmembers of AGOA without repercussions for its quota-free access to the U.S. market.

\footnotetext{
${ }^{7}$ Using the following equation to summarize the CBS's balance sheet: M=NFA+NDA, where M represents base money, and NFA and NDA represent net foreign assets and net domestic assets, respectively.

${ }^{8}$ This sub section does not discuss the benefits deriving from Swaziland's membership in the Southern Africa Development Community (SADC) and the Common Market for Eastern and Southern Africa (COMESA). As yet, these benefits are relatively small.
} 
With relevant U.S. import tariffs at more than 15 percent, ${ }^{9}$ on average, and with foreign inputs accounting for at least 50 percent of total costs, value added in Swaziland is effectively protected at a rate of 30 percent or more. ${ }^{10} 11$

13. Since textile quotas under the Uruguay Round Agreement on Textiles and Clothing (ATC) were lifted at the beginning of 2005, exports by low-cost producers that are no longer quota-constrained, in particular China, have increased. ${ }^{12}$ Producers in Swaziland have reported steep declines in garment exports and unofficial estimates suggest that some 15,000 jobs (about half of the sector's total) have been lost since early 2004.

14. Additional shocks affecting the garment sector are likely to occur:

- The "third party fabric provision" under AGOA is scheduled to be phased out in the fall of 2007. Maintaining tariff-free access to the U.S. market would then require sourcing fiber inputs from AGOA sources - which are generally more expensive than the current inputs, most of which originate in Asia;

- The safeguards imposed by the U.S. and the EU in 2005 on garment imports from China (renewable on an annual basis) are likely to expire by mid-2008. In the case of the U.S., these safeguards limit the annual growth of garment imports from China to 7.5 percent; and

- Multilateral tariff reduction of nonagricultural products under the Doha round is likely to gradually erode the significance of tariff-free market access.

15. Another adverse shock is the imminent change in the EU Sugar Protocol in 2007, implying a reduction in sugar prices of 36 percent over a 4-year period. Also, SPS access may expire in 2006.

\footnotetext{
${ }^{9}$ See USAID (2004).

${ }^{10}$ Thus, for given foreign input costs, the cost of finishing garment products (the "cut, make, and trim" phase) could be at least 30 percent higher in Swaziland than in a country not receiving these preferences, at the same after-tariff import costs.

${ }^{11}$ Since EU access rules do not contain the equivalent of AGOA's "third party fabric provision," garment exports to the EU have been very small.

${ }^{12}$ Under the ATC, which superseded the Multifiber Arrangement (MFA), these bilateral quotas were to be eliminated in four phases over the period 1995-2005 (1995-98; 1998-2002; 2002-04; and the beginning of 2005). The last ATC phase was expected to have the largest impact because it applied to products that were highly quota constrained and that account for the bulk of Swaziland's exports.
} 


\section{Exchange rate developments}

16. Swaziland's external competitiveness is strongly influenced by its currency peg to the South African rand. On the one hand, this arrangement has precluded any exchange rate movement against Swaziland's main trading partner, South Africa. Conditioned by this regime, domestic price movements in Swaziland have been largely similar to those in South Africa.

Figure I.1. Real and Nominal Effective Exchange Rates, Jan 2001-Sep 2005

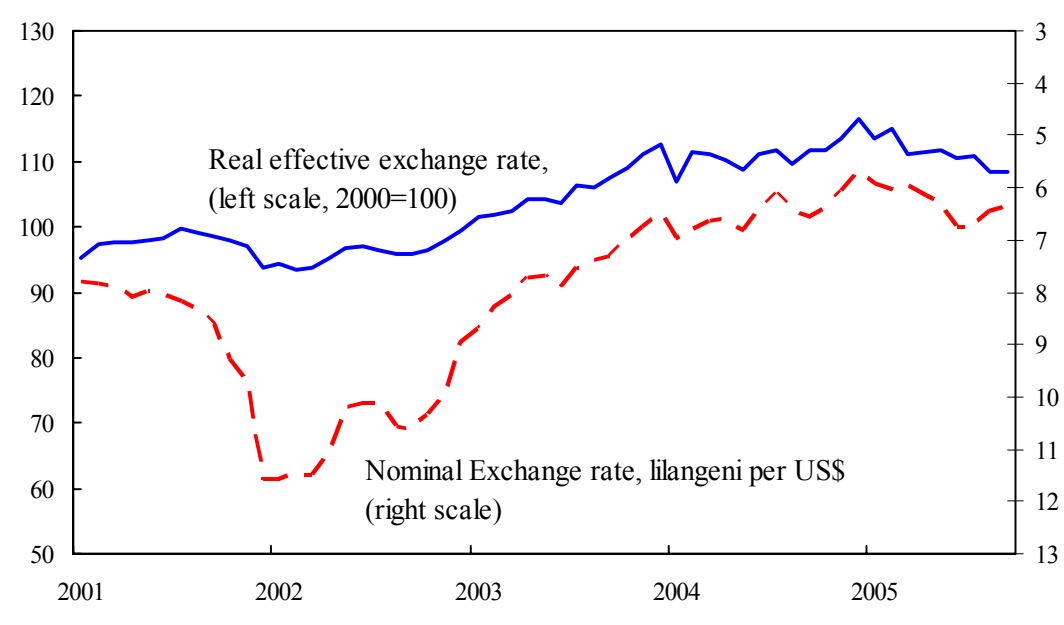

Source: IMF, International Financial Statistics and staff estimates.

17. On the other hand, between 2002 and 2004, the strength of the rand combined with the general depreciation of the U.S. dollar, led to a sizable appreciation of the lilangeni relative to the dollar, by 50 percent in nominal terms and 24 percent in real effective terms (Figure I.1). ${ }^{13}$ Developments in nominal and real effective exchange rates are widely used indicators of overall competitiveness. In the case of Swaziland, profit margins of many Swazi exporters, being price takers in international markets, are likely to have been squeezed by the lilangeni's strong appreciation. ${ }^{14}$ During 2005, the lilangeni weakened against the U.S. dollar by about 13 percent, partly offsetting the earlier appreciation.

\footnotetext{
${ }^{13}$ The real effective exchange rates were calculated on the basis of 2002 data on partner country weights that reflect each country's relative share in Swaziland's trade (imports plus exports), and using Swaziland's consumer price index (CPI). An alternative and more appropriate methodology, given the focus on cost competitiveness, would have been to use unit labor costs, but data limitations prevented this.

${ }^{14}$ The share of the U.S. dollar in Swazi exports is estimated at 35 percent, with about one third of that amount concerning exports to the U.S. However, exporters invoicing in rand would still have experienced a profit squeeze since, in setting prices, their customers would have factored in the lilangeni's appreciation.
} 


\section{Wage costs}

18. Given the openness of Swaziland's economy, its external competitiveness is closely related to its wage level. In addition, the country's fixed exchange rate highlights the importance of domestic cost control for maintaining competitiveness. Available data on minimum wages in manufacturing show an increase of 17.5 percent during 2002-04 (Figure I.2). However, reflecting the appreciation of the lilangeni, the nominal wage expressed in US dollars increased by 58 percent during the period.

Figure I.2. Monthly Minimum Wage in Manufacturing and Processing

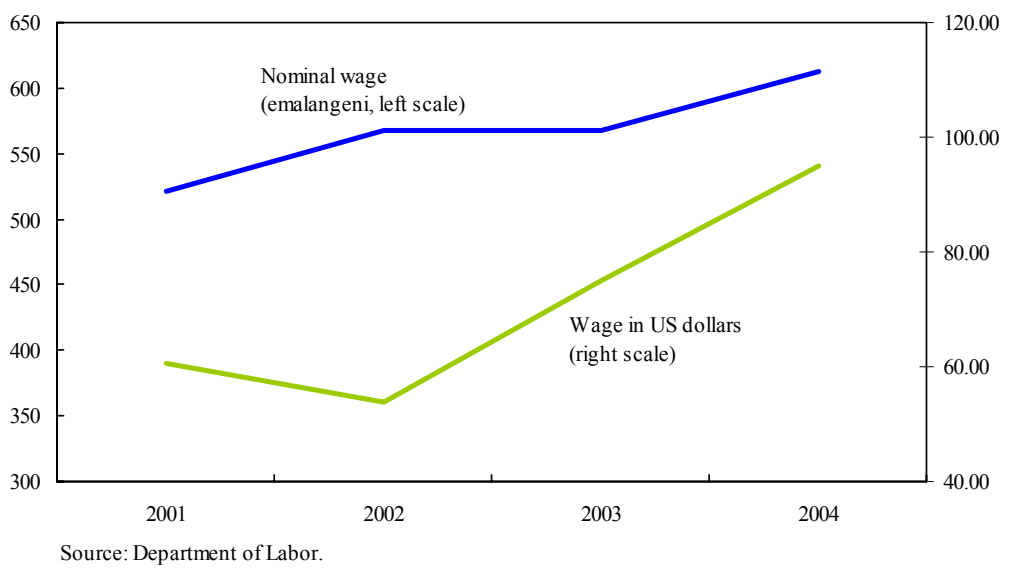

19. Available data, for the garment sector only, indicate that in 2002 Swazi nominal wages were around the level of those in Asian countries, except China, which has a much higher rate. In addition, although lower than in South Africa, Swazi nominal wages were at the same level as those in Lesotho but exceeded those of Kenya and Madagascar.

Figure I.3. Garment Sector Wages for Selected Countries, 2002 (In U.S. dollars per hour)

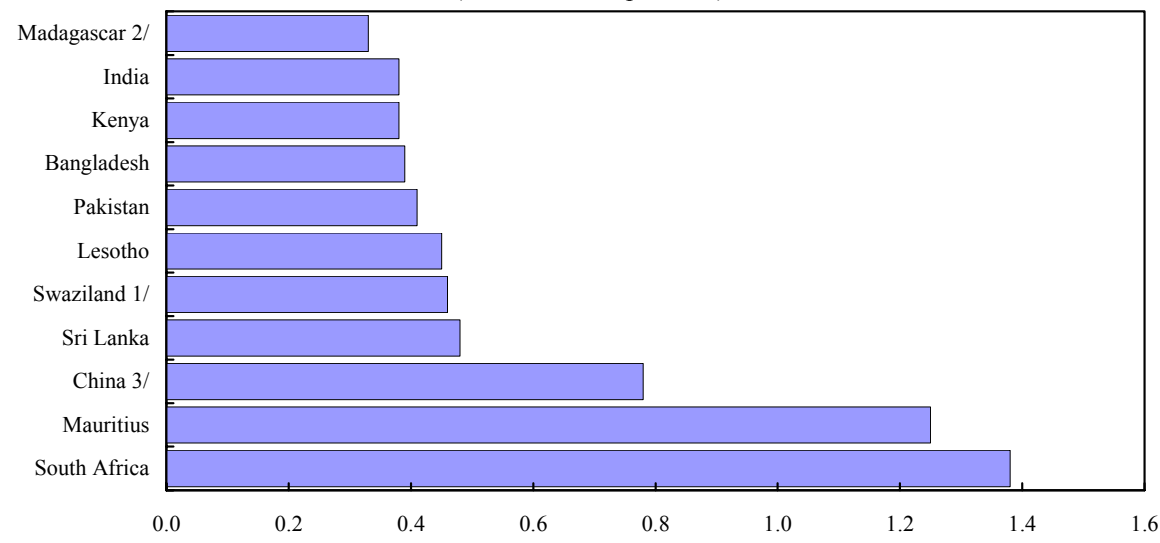

Source : USITC (2004); Cadot and Nasir (2001), USAID (2004). 
20. Although no systematic studies have been conducted on labor productivity in Swaziland, anecdotal evidence suggests that, except for the sugar sector, productivity is relatively low. ${ }^{15}$ Deteriorating social indicators, including increased absenteeism partly due to the HIV/AIDS pandemic, have aggravated the problem.

21. In the agricultural sector, data on yields present a mixed picture. Sugar cane, one of the two main crops, is mainly grown on privately owned, well-irrigated, Title Deed Land (TDL) and output per hectare has increased by some 9 percent since 1998/99 (the Swazi sugar industry is generally considered as efficient and competitive). However, maize, the other main crop, is primarily cultivated on rain-fed communal Swazi Nation Land (accounting for 60 percent of Swazi territory), where output per hectare has almost halved since 1998/99 under the influence of drought, soil erosion, lack of investment and credit, and, increasingly, the effects of the HIV/AIDS pandemic. Productivity declines have affected other food staples as well and since acreage was not increased, absolute declines in food production have occurred, leading to upward pressures on prices.

Figure I.4 Sugarcane and Maize Yield, (In metric tons per hectare)

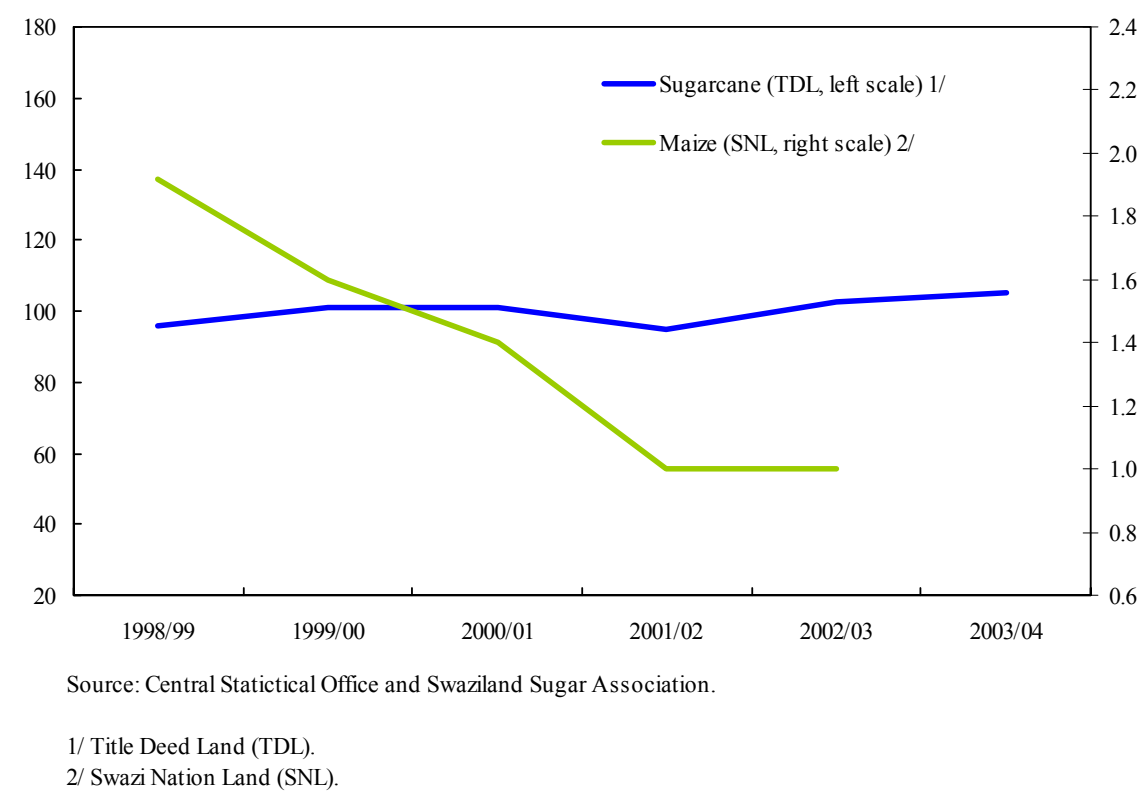

\section{Investment climate}

22. Swaziland's investment climate has been negatively affected by perceptions about poor governance, corruption, high costs of utilities and transportation, and a relatively burdensome regulatory environment. These factors have tended to outweigh the benefits of relatively little government intervention and a low crime rate.

${ }^{15}$ USAID (2004), page 11, refers to a 50 percent higher productivity in Vietnam's garment sector. 
23. Governance issues have had a major impact on the business climate. A rule-oflaw crisis in 2002/03 concerning a property dispute that had negatively affected investors' perceptions has been resolved, contributing to some restoration of confidence. Although a new Constitution, which clarifies various individual rights including provisions to protect private property and provides a clearer separation of the executive, legislature, and the judiciary, became effective in February 2006, uncertainty remains about its implementation. Finally, the lack of important legislation, such as an Investment $\operatorname{Code}^{16}$ and a Companies Act creates uncertainty.

\section{Among CMA countries, Swaziland ranks lowest on Transparency}

International's Corruption Perception Index (Table I. 1) and, on various occasions, highranking government officials, including the Prime Minister, have identified corruption as a major policy challenge.

\begin{tabular}{|c|c|c|c|c|}
\hline \multicolumn{5}{|c|}{ Table I.1. Corruption Perception Index } \\
\hline & $\begin{array}{l}2004 \\
\text { (Mean Value) }\end{array}$ & $\begin{array}{l}2005 \\
\text { (Mean Value) }\end{array}$ & $\begin{array}{r}2004 \\
\text { Ranking } \\
\end{array}$ & $\begin{array}{r}2005 \\
\text { Ranking } \\
\end{array}$ \\
\hline Botswan & 6.0 & 5.9 & 31.0 & 32.0 \\
\hline South & 4.6 & 4.5 & 44.0 & 46.0 \\
\hline Lesoth & 3.4 & 3.4 & $\ldots$ & 70.0 \\
\hline Swaziland & 3.0 & 2.7 & $\ldots$ & 103.0 \\
\hline Africa & 2.9 & 2.8 & & \\
\hline World & 4.2 & 4.2 & & \\
\hline
\end{tabular}

25. Utilities and transportation constitute significant bottlenecks for the Swazi business community. According to a survey conducted by the Southern Africa Global Competitiveness Hub (2004), the cost of utilities is relatively high. Power outages (as well as power surges) occur frequently, and many companies reportedly have back up generators. Telecommunications are expensive and internet service needs to be improved. All utilities are provided by public enterprises that charge relatively high tariffs. Likewise, a survey by

\footnotetext{
${ }^{16}$ Currently, available incentives are scattered in several enactments, and responsibilities are shared among various ministries.
} 
USAID (2004) concluded that despite a relatively well-developed road system and proximity to major seaports such as Durban and Maputo, transportation is costly and unreliable. ${ }^{17}$

26. Administrative procedures are complicated and suffer from a lack of transparency and coordination among government agencies. ${ }^{18}$ For new businesses, the Swaziland Investment Promotion Agency (SIPA), officially a one-stop shop, is often confronted with intervening actions of other government agencies. Approval procedures are time-consuming and lack transparency. Sometimes, rules and regulations are contradictory. ${ }^{19}$ In addition, existing businesses are faced with time-consuming customs procedures, and arbitrary health and sanitary inspections. The perception is widespread that available incentives are granted to nonresidents only and do not apply to local entrepreneurs.

\section{Land tenure issues are being handled by various ministries, which is time} consuming. In addition, there are no established procedures for the purchase of Government Title Deed Land. Single women are allowed to hold property title, but married women may only do so with their husband's consent.

\section{Swaziland's standard corporate tax rate (30 percent) is in line with neighboring} countries. The Minister of Finance may, with Cabinet approval, designate an enterprise as a Development Enterprise and grant tax concessions, implying a reduction of the corporate tax rate to 10 percent (the lowest among SACU members) for ten years and exemption from withholding tax on dividends, also for ten years. However, the requirement that new businesses pay provisional corporate income tax six months after commencing operations constitutes a heavy financial burden. In addition, in the absence of a tax tribunal, tax disputes are handled, with long delays, by the judicial courts.

\section{Conclusion}

29. Available evidence indicates that Swaziland's external competitiveness has deteriorated in recent years due to erosion of trade preferences, exchange rate appreciation, relatively low labor productivity, partly because of HIV/AIDS, and a poor investment climate. In 2005, real GDP growth slowed to less than 2 percent and gross international reserves declined to 1.1 months of imports at end-September 2005. ${ }^{20}$ Given Swaziland's

${ }^{17}$ The cost of shipping a container from Hong Kong to Durban averaged between 2,500-3,000 rand. From Durban to Swaziland, the cost of inland transport averaged between 7,000-10,000 rand.

${ }^{18}$ Paragraphs 26-28 are mainly based on Southern Africa Global Competitiveness Hub (2004).

${ }^{19}$ For instance, an investor needs a Trading License to get an Entry (Work) Permit, which can only be obtained if he has a Trading License.

${ }^{20}$ Another reason for the decline in Swaziland's international reserves relates to the domestic financing of fiscal deficits (see next chapter). 
participation in the CMA, i.e., it has no control over the exchange rate and does not have an independent monetary policy, effective implementation of prudent fiscal policies and bold structural reform measures would go a long way to improve Swaziland's external competitiveness. 


\section{References}

Cadott, Olivier, and John Nassir, 2001, "Incentives and Obstacles to Growth: Lessons from Manufacturing Case Studies in Madagascar", World Bank, RPED Discussion Paper No.117.

Humpage, Owen F. and Jean M. McIntire, 1995 "An Introduction to Currency Boards," Federal Reserve Bank of Cleveland Economic Review (31), p.2-11.

Masson, Paul R., and Catherine Patillo, 2005, The Monetary Geography of Africa, (Washington D.C., Brookings Institutions Press).

Southern Africa Global Competitiveness Hub, 2005, "The Swaziland Investor Road Map."

United States Agency for International Development (USAID), 2004, "the Elimination of Quotas Under the World Trade Organization Agreement on Textile and Clothing: The Impact on Swaziland," (Botswana: USAID/Regional Center for Southern Africa).

United States International Trade Commission (USITC), 2004, Textiles and Apparel: "Assessment of the Competitiveness of Certain Foreign Suppliers to the U.S. Market," USTIC Publication No. 3671 (Washington, DC). 


\section{Appendix: CMA features}

30. The CMA's broad objectives are to confer the advantages of a common monetary area on its members, provide for the sustained economic development of the CMA as a whole, encourage the advancement of the less developed members, and afford to all parties equitable benefits arising from the maintenance and development of the CMA (Article 2).

31. Article 2 of the CMA agreement gives the three small member countries (Lesotho, Namibia, and Swaziland) the right to issue national currencies, and their bilateral agreements with South Africa define the areas in which their currencies are legal tender. The local currencies (in Swaziland, the lilangeni) issued by the three members are legal tender in their respective countries only. The South African rand, however, is legal tender throughout the CMA.

32. Under the terms of the CMA Agreement (Article 3), no restrictions can be imposed on the transfer of funds, whether for current or capital transactions, to or from any member country. The only exceptions result from the member countries' investment or liquidity requirements prescribed for financial institutions. The small member countries tend to view the investment and liquidity requirements as a measure of savings mobilization for development purposes. The regulations requiring the investment of funds by financial institutions in domestic securities or credits to local businesses or individuals are, in effect, minimum local asset requirements. These regulations are meant to address the concerns of the three small, less developed, CMA members that funds generated in their territories and deposited with local financial institutions tended to flow to the more developed capital markets of South Africa. Swaziland's minimum local assets to total deposits ratio, which has been reduced in recent years, is 13 percent at present.

33. The CMA Agreement provides for the three small member countries to have access to the South African capital and money markets, but only through prescribed investments or approved securities that can be held by financial institutions in South Africa.

The CMA Agreement (Article 5) requires the smaller countries' exchange control regulations to be - in all material aspects - similar to those in effect in South Africa.

34. Since the rand is legal tender in all CMA countries (but the currencies of the three small CMA members are not legal tender in South Africa), South Africa compensates them for forgone seigniorage. Compensation is based on a formula equal to the product of (i) two-thirds of the annual yield on the most recently issued long-term South African government stock; and (ii) the volume of rand estimated to be in circulation in the member country concerned. The ratio of two-thirds was established on the assumption that it approximated the yield of a portfolio of reserve assets comprising both long-term and shortterm maturities, assuming that the average yield would be less than the full long-term yield.

35. To facilitate the implementation of the CMA Agreement, the member countries have established a commission in which each of them has one representative (along with some 
advisors, as needed). The commission holds regular consultations - at least once a yearwith the aim of reconciling the interests of member countries on common issues pertaining to monetary and foreign exchange policies. It also convenes at other times at the request of a member country. Article 9 of the CMA Agreement provides for the establishment of a tribunal to arbitrate disputes that might arise between member countries regarding the interpretation or application of the agreement. 
Table 1. Swaziland: Gross Domestic Product by Sector of Origin at 2000 Constant Prices, 2000-2004

\begin{tabular}{|c|c|c|c|c|c|}
\hline & 2000 & 2001 & 2002 & 2003 & $\begin{array}{r}2004 \\
\text { Est. }\end{array}$ \\
\hline & \multicolumn{5}{|c|}{ (In millions of emalangeni) } \\
\hline Primary production & $1,081.1$ & 983.1 & $1,038.3$ & $1,074.8$ & $1,072.2$ \\
\hline Agriculture and livestock & 985.9 & 894.8 & 945.3 & 987.9 & 978.2 \\
\hline SNL crops $1 /$ & 80.4 & 72.9 & 57.8 & 49.7 & 66.3 \\
\hline TDL crops $2 /$ & 790.4 & 747.6 & 803.8 & 855.0 & 828.2 \\
\hline Livestock and other & 115.1 & 74.3 & 83.7 & 83.2 & 83.7 \\
\hline Forestry & 53.0 & 54.5 & 55.4 & 56.8 & 61.2 \\
\hline Mining & 42.2 & 33.8 & 37.6 & 30.0 & 32.7 \\
\hline Secondary production & $2,954.8$ & $3,050.7$ & $3,123.5$ & $3,176.2$ & $3,211.5$ \\
\hline Manufacturing & $2,400.2$ & $2,422.8$ & $2,474.9$ & $2,519.8$ & $2,533.3$ \\
\hline Electricity and water & 105.9 & 111.7 & 127.5 & 130.5 & 127.3 \\
\hline Construction & 448.7 & 516.2 & 521.0 & 525.9 & 550.9 \\
\hline Services & $2,660.5$ & $2,737.7$ & $2,810.9$ & $2,877.9$ & $2,965.4$ \\
\hline Wholesale and retail & 494.4 & 530.2 & 557.9 & 592.0 & 622.2 \\
\hline Hotels and restaurants & 122.9 & 115.9 & 120.8 & 124.5 & 150.1 \\
\hline Transport & 218.1 & 216.7 & 223.0 & 252.8 & 267.1 \\
\hline Communications & 140.7 & 153.5 & 156.3 & 122.3 & 130.7 \\
\hline Banking, finance, and insurance & 248.4 & 243.0 & 266.6 & 293.9 & 357.5 \\
\hline Real estate & 81.4 & 83.8 & 86.9 & 89.5 & 94.2 \\
\hline Government services & $1,243.5$ & $1,268.4$ & $1,281.0$ & $1,319.6$ & $1,334.8$ \\
\hline Other services & 80.7 & 82.3 & 83.6 & 85.5 & 87.5 \\
\hline Owner-occupied dwellings & 198.1 & 207.9 & 214.6 & 212.5 & 206.4 \\
\hline Imputed bank service charge & -167.7 & -164.1 & -179.8 & -214.9 & -285.0 \\
\hline GDP at factor cost & $6,696.4$ & $6,771.4$ & $6,972.7$ & $7,128.9$ & $7,249.0$ \\
\hline Indirect taxes less subsidies & $2,941.2$ & $3,021.4$ & $3,102.6$ & $3,192.9$ & $3,285.3$ \\
\hline \multirow[t]{2}{*}{ GDP at market prices ${ }^{3 /}$} & $9,637.6$ & $9,792.8$ & $10,075.3$ & $10,321.7$ & $10,534.3$ \\
\hline & \multicolumn{5}{|c|}{ (Annual percentage change) } \\
\hline Agriculture and livestock & 1.1 & -9.2 & 5.6 & 4.5 & -1.0 \\
\hline Forestry & 2.3 & 2.8 & 1.6 & 2.7 & 7.8 \\
\hline Mining & -22.8 & -19.9 & 11.2 & -20.1 & 9.0 \\
\hline Manufacturing & 1.5 & 0.9 & 2.1 & 1.8 & 0.5 \\
\hline Electricity and water & -6.7 & 5.4 & 14.2 & 2.3 & -2.5 \\
\hline Construction & 9.8 & 15.0 & 0.9 & 0.9 & 4.7 \\
\hline Services & 4.4 & 2.9 & 2.7 & 2.4 & 3.0 \\
\hline \multirow[t]{2}{*}{ GDP at market prices } & 2.6 & 1.6 & 2.9 & 2.4 & 2.1 \\
\hline & \multicolumn{5}{|c|}{ (In percent of GDP at factor cost) } \\
\hline Agriculture and livestock & 14.7 & 13.2 & 13.6 & 13.9 & 13.5 \\
\hline Forestry & 0.8 & 0.8 & 0.8 & 0.8 & 0.8 \\
\hline Mining & 0.6 & 0.5 & 0.5 & 0.4 & 0.5 \\
\hline Manufacturing & 35.8 & 35.8 & 35.5 & 35.3 & 34.9 \\
\hline Electricity and water & 1.6 & 1.6 & 1.8 & 1.8 & 1.8 \\
\hline Construction & 6.7 & 7.6 & 7.5 & 7.4 & 7.6 \\
\hline Services & 39.7 & 40.4 & 40.3 & 40.4 & 40.9 \\
\hline
\end{tabular}

Source: Central Statistical Office.

1/ Swazi Nation Land (SNL).

2/ Title Deed Land (TDL).

3/ Under review by the CSO; data on indirect taxes used for estimation of GDP may contain errors and are subject to downward

revisions based on the review.

\section{CInternational Monetary Fund. Not for Redistribution}


Table 2. Swaziland: Gross Domestic Product by Sector of Origin at Current Prices, 2000-2004 (In millions of emalangeni, unless otherwise indicated)

\begin{tabular}{|c|c|c|c|c|c|}
\hline & 2000 & 2001 & 2002 & 2003 & $\begin{array}{r}2004 \\
\text { Est. }\end{array}$ \\
\hline Primary production & $1,081.1$ & $1,013.2$ & $1,122.3$ & $1,100.9$ & $1,120.6$ \\
\hline Agriculture and livestock & 985.9 & 922.8 & $1,018.4$ & 993.1 & $1,001.8$ \\
\hline SNL crops $1 /$ & 80.4 & 92.1 & 70.8 & 74.1 & 103.2 \\
\hline TDL crops $2 /$ & 790.4 & 752.0 & 859.1 & 838.6 & 816.5 \\
\hline Livestock and other & 115.1 & 78.7 & 88.5 & 80.4 & 82.1 \\
\hline Forestry & 53.0 & 55.8 & 56.6 & 57.4 & 61.8 \\
\hline Mining & 42.2 & 34.6 & 47.3 & 50.4 & 57.0 \\
\hline Secondary production & $2,954.8$ & $3,273.8$ & $3,615.0$ & $3,939.3$ & $4,334.3$ \\
\hline Manufacturing & $2,400.2$ & $2,710.1$ & $2,983.3$ & $3,269.7$ & $3,403.0$ \\
\hline Electricity and water & 105.9 & 111.7 & 127.5 & 130.6 & 134.0 \\
\hline Construction & 448.7 & 452.0 & 504.2 & 539.0 & 797.3 \\
\hline Services & $2,660.5$ & $2,847.3$ & $3,138.4$ & $3,470.5$ & $3,770.4$ \\
\hline Wholesale and retail & 494.4 & 563.6 & 642.5 & 715.7 & 797.3 \\
\hline Hotels and restaurants & 122.9 & 125.6 & 143.2 & 146.8 & 147.8 \\
\hline Transport & 218.1 & 223.2 & 248.7 & 267.7 & 261.1 \\
\hline Communications & 140.7 & 161.8 & 186.1 & 214.0 & 218.6 \\
\hline Banking, finance, and insurance & 248.4 & 242.3 & 285.1 & 314.2 & 382.4 \\
\hline Real estate & 81.4 & 83.6 & 86.3 & 89.4 & 93.2 \\
\hline Government services & $1,243.5$ & $1,363.4$ & $1,496.1$ & $1,687.3$ & $1,856.0$ \\
\hline Other services & 80.7 & 82.6 & 85.0 & 86.7 & 89.2 \\
\hline Owner-occupied dwellings & 198.1 & 164.9 & 158.0 & 161.0 & 165.7 \\
\hline Imputed bank service charge & -167.7 & -163.7 & -192.6 & -212.3 & -240.9 \\
\hline GDP at factor cost & $6,696.4$ & $7,134.3$ & $7,875.7$ & $8,510.7$ & $9,225.3$ \\
\hline Indirect taxes less subsidies & $2,941.2$ & $3,711.8$ & $4,684.3$ & $5,911.7$ & $7,037.0$ \\
\hline GDP at market prices ${ }^{3 /}$ & $9,637.6$ & $10,846.1$ & $12,560.0$ & $14,422.4$ & $16,262.3$ \\
\hline \multicolumn{6}{|l|}{ Memorandum items: } \\
\hline GDP deflator (index, $2000=100$ ) & 100.0 & 110.8 & 124.7 & 139.7 & 154.4 \\
\hline (percent change) & 11.6 & 10.8 & 12.6 & 12.1 & 10.5 \\
\hline
\end{tabular}

Source: Central Statistical Office.

1/ Swazi Nation Land (SNL).

2/ Title Deed Land (TDL).

3/ Under review by the CSO; data on indirect taxes used for estimation of GDP may contain errors and are subject to downward revisions based on the review. 
Table 3. Swaziland: Gross Domestic Product by Expenditure Category at Current Prices, 2000-2004

\begin{tabular}{|c|c|c|c|c|c|}
\hline & 2000 & 2001 & 2002 & 2003 & $\begin{array}{r}2004 \\
\text { Est. }\end{array}$ \\
\hline & \multicolumn{5}{|c|}{ (In millions of emalangeni) } \\
\hline Final consumption expenditure & $9,344.0$ & $10,515.1$ & $10,107.0$ & $11,880.8$ & $13,975.0$ \\
\hline Private & $7,580.7$ & $8,592.5$ & $7,772.3$ & $9,199.8$ & $10,552.4$ \\
\hline Government & $1,763.3$ & $1,922.6$ & $2,334.7$ & $2,681.0$ & $3,422.6$ \\
\hline Capital formation & $1,793.9$ & $1,996.4$ & $2,486.4$ & $2,590.4$ & $2,831.6$ \\
\hline Gross fixed capital formation & $1,793.9$ & $1,996.4$ & $2,486.4$ & $2,590.4$ & $2,831.6$ \\
\hline Public & $1,189.5$ & $1,129.3$ & $1,550.9$ & $1,772.6$ & $1,572.6$ \\
\hline Private & 604.4 & 867.1 & 935.5 & 817.8 & $1,259.0$ \\
\hline Increases in stocks & 0.0 & 0.0 & 0.0 & 0.0 & 0.0 \\
\hline Balance of trade for goods and nonfactor services & $-1,500.3$ & $-1,665.4$ & -33.4 & -48.8 & -544.3 \\
\hline Exports & $7,863.2$ & $9,954.1$ & $11,923.0$ & $12,416.1$ & $15,223.1$ \\
\hline Imports, f.o.b. $1 /$ & $-9,363.5$ & $-11,619.5$ & $-11,956.4$ & $-12,464.9$ & $-15,767.4$ \\
\hline \multirow[t]{2}{*}{ GDP at market prices ${ }^{2 /}$} & $9,637.6$ & $10,846.1$ & $12,560.0$ & $14,422.4$ & $16,262.3$ \\
\hline & \multicolumn{5}{|c|}{ (In percent of GDP) } \\
\hline Final consumption expenditure & 97.0 & 96.9 & 80.5 & 82.4 & 85.9 \\
\hline Private & 78.7 & 79.2 & 61.9 & 63.8 & 64.9 \\
\hline Government & 18.3 & 17.7 & 18.6 & 18.6 & 21.0 \\
\hline Capital formation & 18.6 & 18.4 & 19.8 & 18.0 & 17.4 \\
\hline Gross fixed capital formation & 18.6 & 18.4 & 19.8 & 18.0 & 17.4 \\
\hline Public & 12.3 & 10.4 & 12.3 & 12.3 & 9.7 \\
\hline Private & 6.3 & 8.0 & 7.4 & 5.7 & 7.7 \\
\hline Increases in stocks & 0.0 & 0.0 & 0.0 & 0.0 & 0.0 \\
\hline Balance of trade for goods and nonfactor services & -15.6 & -15.4 & -0.3 & -0.3 & -3.3 \\
\hline Exports of goods and services & 81.6 & 91.8 & 94.9 & 86.1 & 93.6 \\
\hline Imports of goods and services, f.o.b. & -97.2 & -107.1 & -95.2 & -86.4 & -97.0 \\
\hline GDP at market prices & 100.0 & 100.0 & 100.0 & 100.0 & 100.0 \\
\hline
\end{tabular}

Source: Central Statistical Office.

1/ All Southern African Customs Union receipts are treated as indirect taxes (and therefore deducted from imports, c.i.f. to get imports, f.o.b.), accounting for the significant difference between the trade figures in the national income accounts reported in this table and those in the balance of payments.

2/ Under review by the CSO; data on indirect taxes used for estimation of GDP may contain errors and are subject to downward revisions based on the review. 
Table 4. Swaziland: Population and Labor Force Estimates, 2000-2003

\begin{tabular}{|c|c|c|c|c|}
\hline & 2000 & 2001 & 2002 & 2003 \\
\hline & \multicolumn{4}{|c|}{ (In percent, unless otherwise indicated) } \\
\hline Annual population growth $1 /$ & 2.1 & 2.0 & -0.5 & 0.0 \\
\hline Population (in thousands) $1 /$ & $1,029.6$ & $1,050.0$ & $1,045.0$ & $1,045.0$ \\
\hline \multirow[t]{2}{*}{ Population density (per sq.km.) } & 59.3 & 60.5 & 60.2 & 60.2 \\
\hline & \multicolumn{4}{|c|}{ (In thousands, unless otherwise indicated) } \\
\hline Labor force $2 /$ & 383.0 & 394.0 & $\ldots$ & $\ldots$ \\
\hline Total employment & 259.1 & 263.1 & $\ldots$ & $\ldots$ \\
\hline Formal employment 3/ & 92.0 & 93.0 & 95.0 & 102.0 \\
\hline Public sector & 29.0 & 29.0 & 30.0 & 30.0 \\
\hline Private sector & 63.0 & 64.0 & 65.0 & 72.0 \\
\hline Informal employment & 167.1 & 170.1 & $\ldots$ & $\ldots$ \\
\hline Unemployment rate (in percent) & 31.3 & 31.3 & 30.0 & 29.0 \\
\hline
\end{tabular}

Sources: Central Statistical Office; World Bank; United Nations; and staff estimates.

1/ Estimate, United Nations and national authorities.

2/ World Bank estimates.

3/ Central Statistical Office data. 
Table 5. Swaziland: Developments in Crop Production, 2000/01-2004/05 1/

\begin{tabular}{|c|c|c|c|c|c|c|}
\hline & $2000 / 01$ & $2001 / 02$ & $2002 / 03$ & $2003 / 04$ & $\begin{array}{c}2005 / 06 \\
\text { Proj. }\end{array}$ & $2004 / 05$ \\
\hline & \multicolumn{5}{|c|}{ (In thousands of metric tons) } & \\
\hline \multicolumn{7}{|l|}{ Volume } \\
\hline Sugarcane & $4,442.0$ & $4,179.0$ & $4,609.0$ & $5,046.0$ & $4,884.0$ & 4834.0 \\
\hline Cotton & 6.0 & 3.9 & 1.2 & 3.2 & & $\ldots$ \\
\hline Maize & 86.7 & 67.7 & 69.3 & 71 & & $\ldots$ \\
\hline SNL 2/ & 78.8 & 62.0 & 62.4 & $\ldots$ & & $\ldots$ \\
\hline TDL 3/ & 7.9 & 5.7 & 6.9 & $\ldots$ & & $\ldots$ \\
\hline Citrus fruit & 102.3 & 89.8 & 74.4 & 70.9 & & $\ldots$ \\
\hline Tobacco & 0.072 & 0.1 & $\ldots$ & $\ldots$ & & $\ldots$ \\
\hline \multicolumn{7}{|c|}{ (In metric tons per hectare) } \\
\hline \multicolumn{7}{|l|}{ Yield } \\
\hline Sugarcane & 102.0 & 95.0 & 102.0 & 105.0 & 98.0 & 98.2 \\
\hline Cotton & 625.0 & 370.0 & 348.9 & $\ldots$ & & $\ldots$ \\
\hline Maize & & & & & & $\ldots$ \\
\hline SNL 2/ & 1.4 & 1.0 & 1.0 & $\ldots$ & & $\ldots$ \\
\hline TDL 3/ & 1.4 & 0.9 & $\ldots$ & $\ldots$ & & $\ldots$ \\
\hline Citrus fruit & 40.9 & 40.6 & $\ldots$ & $\ldots$ & & $\ldots$ \\
\hline \multicolumn{7}{|c|}{ (In millions of emalangeni) } \\
\hline \multicolumn{7}{|l|}{ Value } \\
\hline Sugarcane & $5,399.3$ & $5,887.0$ & $\ldots$ & $\ldots$ & & $\ldots$ \\
\hline Cotton & 31.6 & 9.8 & $\ldots$ & $\ldots$ & & $\ldots$ \\
\hline Maize & 76.5 & 51.6 & $\ldots$ & $\ldots$ & & $\ldots$ \\
\hline SNL 2/ & 70.9 & 46.5 & $\ldots$ & $\ldots$ & & $\ldots$ \\
\hline TDL $3 /$ & 5.6 & 5.1 & $\ldots$ & $\ldots$ & & $\ldots$ \\
\hline Citrus fruit & 112.0 & 146.6 & $\ldots$ & $\ldots$ & & $\ldots$ \\
\hline \multicolumn{7}{|c|}{ (In emalangeni per metric ton) } \\
\hline Producer prices & & & & & & \\
\hline Sugarcane & $1,215.5$ & $1,408.7$ & $\ldots$ & $\ldots$ & & $\ldots$ \\
\hline Cotton & $2,412.2$ & $2,500.0$ & $\ldots$ & $\ldots$ & & $\ldots$ \\
\hline Maize & 900.0 & 750.0 & $\ldots$ & $\ldots$ & & $\ldots$ \\
\hline Citrus fruit & $1,095.0$ & $1,632.0$ & $\ldots$ & $\ldots$ & & $\ldots$ \\
\hline
\end{tabular}

Source: Central Statistical Office and Swaziland Sugar Association.

1/ Variable crop years.

2/ Swazi Nation Land (SNL).

3/ Title Deed Land (TDL). 
Table 6. Swaziland: Developments in Sugar Production, Processing, and Consumption, 2000/01-2004/05

\begin{tabular}{|c|c|c|c|c|c|}
\hline & $2000 / 01$ & $2001 / 02$ & $2002 / 03$ & $2003 / 04$ & $2004 / 05$ \\
\hline & \multicolumn{5}{|c|}{ (In thousands of hectares) } \\
\hline \multicolumn{6}{|l|}{ Crop area } \\
\hline Area under cultivation & 45.0 & 47.0 & 48.0 & 49.9 & 50.9 \\
\hline Area harvested & 44.0 & 44.0 & 45.0 & 48.0 & 49.7 \\
\hline & \multicolumn{5}{|c|}{ (In thousands of metric tons) } \\
\hline \multicolumn{6}{|l|}{ Production and processing } \\
\hline Cane & $4,442.0$ & $4,179.0$ & $4,609.0$ & $5,046.0$ & $4,884.0$ \\
\hline Sucrose & 603.0 & 573.0 & 666.0 & 719.0 & 686.0 \\
\hline \multirow[t]{2}{*}{ Sugar } & 528.0 & 501.0 & 583.0 & 628.0 & 598.0 \\
\hline & \multicolumn{5}{|c|}{ (In metric tons per hectare harvested) } \\
\hline Sugarcane yield & 102.0 & 95.0 & 102.0 & 105.0 & 98.0 \\
\hline & \multicolumn{5}{|c|}{ (In thousands of metric tons) } \\
\hline Domestic consumption and local sales $3 /$ & 255.0 & 296.0 & 281.0 & 332.0 & 311.0 \\
\hline
\end{tabular}

Source: Swaziland Sugar Association.

1/ Sugarcane is grown mainly on Title Deed Land.

2/ Crop year beginning in April, unless otherwise indicated.

3/ Calendar year. "Domestic" covers Swaziland, as well as the other members of the Southern African Customs Union. 
Table 7. Swaziland: Mineral Production and Exports, 2000-2004 (In thousands of metric tons)

\begin{tabular}{|c|c|c|c|c|c|c|}
\hline & 1999 & 2000 & 2001 & 2002 & 2003 & 2004 \\
\hline \multicolumn{7}{|l|}{ Production } \\
\hline Chrysolite asbestos $1 /$ & 22.9 & 12.7 & 3.0 & $\ldots$ & $\ldots$ & $\ldots$ \\
\hline Coal & 426.3 & 378.0 & 313.0 & 553.4 & 448.7 & 448.3 \\
\hline \multicolumn{7}{|l|}{ Exports } \\
\hline Chrysolite asbestos & 23.0 & 13.0 & 3.0 & $\ldots$ & $\ldots$ & $\ldots$ \\
\hline Coal & 426.0 & 378.0 & 313.0 & 553.4 & 355.9 & $\ldots$ \\
\hline
\end{tabular}

Sources: Central Statistical Office; and Geological Survey and Mines Department.

1/ Asbestos mining stopped in 2001. 
Table 8. Swaziland: Index of Industrial Production, 2001-2003

$$
(1985=100)
$$

\begin{tabular}{lrrr}
\hline & 2001 & 2002 & 2003 \\
& & & \\
\hline Mining & 76.6 & 85.6 & 67.9 \\
& & & \\
Manufacturing & 154.2 & 168.4 & 181.4 \\
$\quad$ Sugar refining & 130.9 & 127.2 & 125.5 \\
Other agro-processing & $2,926.7$ & $3,407.2$ & $3,289.3$ \\
Drink processing & 109.1 & 112.6 & 102 \\
Wood pulp, timber, and packaging & 633.1 & 696.1 & 745.6 \\
$\quad$ Other manufacturing & & & \\
& 266.2 & 306.6 & 313.0 \\
All industries & & & \\
\hline
\end{tabular}

Source: Central Statistical Office. 
Table 9. Swaziland: Electrical Power Generation and Demand, 2000-2004 (In millions of kilowatt-hours)

\begin{tabular}{lrrrrr}
\hline & 2000 & 2001 & 2002 & 2003 & 2004 \\
& & & & & \\
& & & & & \\
Total local production and imports 1/ & 812.1 & 846.9 & 893.0 & 994.1 & $1,020.3$ \\
$\quad$ Local production 2/ & 264.7 & 205.6 & 203.7 & 123.0 & 103.5 \\
$\quad$ Hydroelectric & 263.4 & 243.9 & 202.2 & 121.6 & $\ldots$ \\
$\quad$ Diesel & 1.3 & 1.7 & 1.5 & 1.4 & $\ldots$ \\
$\quad$ Imports (from ESKOM) 3/ & 547.4 & 601.3 & 689.3 & 871.1 & 916.8 \\
& & & & & \\
Total sales 1/ & 695.6 & 719.5 & 764.6 & 831.8 & 840.8 \\
$\quad$ Household & 166.9 & 170.8 & 177.8 & 189.1 & 190.6 \\
$\quad$ Commercial & 74.6 & 74.9 & 80.7 & 87.4 & 91.5 \\
Irrigation power and bulk & 454.1 & 473.8 & 506.1 & 555.3 & 558.7 \\
& & & & & \\
\hline
\end{tabular}

Source: Swaziland Electricity Board.

$1 /$ The difference between production and sales is accounted for by transmission and distribution losses.

2/ Excludes self-generated power of several industrial concerns.

3/ South African Electricity Supply Commission (ESKOM). 
Table 10. Swaziland: Retail Price Indices of Urban Families, 2001-2005 1/

\begin{tabular}{|c|c|c|c|c|c|}
\hline & 2001 & 2002 & 2003 & 2004 & $\begin{array}{l}2005 \\
\text { Sept. }\end{array}$ \\
\hline & \multicolumn{5}{|c|}{$($ January $1996=100)$} \\
\hline All-groups index 2/ & 154.1 & 167.9 & 179.8 & 185.6 & 194.5 \\
\hline Food & 151.6 & 177.9 & 198.8 & 210.0 & 227.5 \\
\hline Alcohol and tobacco & 185.4 & 194.3 & 220.5 & 234.2 & 257.2 \\
\hline Clothing and footwear & 130.8 & 135.2 & 143.3 & 144.4 & 146.3 \\
\hline Housing & 127.7 & 127.7 & 137.6 & 144.7 & 146.5 \\
\hline Fuel and power & 276.6 & 282.4 & 303.4 & 317.5 & 328.6 \\
\hline Furniture and appliances & 143.5 & 156.3 & 160.6 & 159.4 & 159.2 \\
\hline Household operations & 180.6 & 192.1 & 202.5 & 208.7 & 209.5 \\
\hline Health care & 149.2 & 153.9 & 162.3 & 161.3 & 163.3 \\
\hline Transport and communications & 162.9 & 172.5 & 177.4 & 181.1 & 199.0 \\
\hline Leisure & 133.6 & 138.4 & 142.9 & 141.3 & 135.8 \\
\hline Education & 193.0 & 254.6 & 272.1 & 278.8 & 313.3 \\
\hline Personal care & 166.6 & 156.7 & 154.4 & 155.4 & 154.2 \\
\hline Miscellaneous & 121.3 & 121.8 & 125.5 & 125.9 & 126.1 \\
\hline Low-income index $3 /$ & 163.8 & 177.9 & 193.3 & 201.3 & 212.3 \\
\hline \multirow[t]{2}{*}{ Middle- and high-income index 4/ } & 153.9 & 167.5 & 179.2 & 185.0 & 193.8 \\
\hline & \multicolumn{5}{|c|}{ (Percentage change over previous year) } \\
\hline All-groups index $2 /$ & 10.8 & 11.5 & 4.6 & 3.2 & 5.0 \\
\hline Low-income index $3 /$ & 9.7 & 12.3 & 4.8 & 4.1 & 5.4 \\
\hline Middle- and high-income index 4/ & 10.8 & 11.5 & 4.7 & 3.2 & 5.0 \\
\hline \multicolumn{6}{|l|}{ Memorandum item: } \\
\hline Consumer price index in South Africa & 4.8 & 12.4 & 0.2 & 3.4 & 4.4 \\
\hline
\end{tabular}

Source: Central Statistical Office.

1/ For 2005, latest available month.

2/ End of period; derived independently from the two income indices on the basis of a different basket of goods and services.

3/ End of period; families with incomes of E 2,399 per annum and below.

4/ End of period; families with incomes between E 2,400 and E 8,760 per annum. 
Table 11. Swaziland: Minimum Wages for General Workers in Selected Industries, 2001-2003 ${ }^{1 /}$

\begin{tabular}{|c|c|c|c|}
\hline & 2001 & 2002 & 2003 \\
\hline & \multicolumn{3}{|c|}{ (In emalangeni per month) } \\
\hline \multicolumn{4}{|l|}{ Retail and wholesale } \\
\hline Mbabane and Manzini & 833.0 & 833.0 & 833.0 \\
\hline Elsewhere & 726.6 & 726.6 & 726.6 \\
\hline & \multicolumn{3}{|c|}{ (In emalangeni per week) } \\
\hline \multirow[t]{2}{*}{ Manufacturing and processing } & 120.3 & 131.1 & 131.1 \\
\hline & \multicolumn{3}{|c|}{ (In emalangeni per day) } \\
\hline \multirow[t]{2}{*}{ Agriculture } & 12.8 & 12.8 & 12.8 \\
\hline & \multicolumn{3}{|c|}{ (In emalangeni per hour) } \\
\hline Building and construction & 4.1 & 4.1 & 4.1 \\
\hline \multirow[t]{2}{*}{ Motor and engineering trades } & 3.3 & 3.3 & 3.3 \\
\hline & \multicolumn{3}{|c|}{ (Annual percentage change) } \\
\hline \multicolumn{4}{|l|}{ Retail and wholesale } \\
\hline Mbabane and Manzini & 8.0 & 0.0 & 0.0 \\
\hline Elsewhere & 8.0 & 0.0 & 0.0 \\
\hline Manufacturing and processing & 8.5 & 9.0 & 0.0 \\
\hline Agriculture & 19.1 & 0.0 & 0.0 \\
\hline Building and construction & 19.5 & 0.0 & 0.0 \\
\hline Motor and engineering trades & 14.2 & 0.0 & 0.0 \\
\hline
\end{tabular}

Source: Department of Labor.

1/ As of December of each year. 
Table 12. Swaziland: Paid Employment in the Private and Public Sectors by Industry, 2000-2003 1/

(In number of employees)

\begin{tabular}{|c|c|c|c|c|c|c|c|c|}
\hline & \multicolumn{2}{|c|}{2000} & \multicolumn{2}{|c|}{2001} & \multicolumn{2}{|c|}{2002} & \multicolumn{2}{|c|}{2003} \\
\hline & Private & Public & Private & Public & Private & Public & Private & Public \\
\hline Agriculture/forestry & 18,425 & 2,159 & 18,470 & 285 & 20,787 & 2,302 & 23,856 & 2,386 \\
\hline Mining and quarrying & 922 & 0 & 620 & 0 & 425 & 0 & 955 & 0 \\
\hline Manufacturing & 18,897 & 0 & 19,898 & 0 & 19,370 & 0 & 20,165 & 0 \\
\hline Electricity and water & 0 & 1,407 & 0 & 1,409 & 0 & 1,455 & 0 & 1,508 \\
\hline Construction & 3,718 & 1,795 & 3,968 & 1,811 & 4,115 & 1,982 & 3,785 & 2,055 \\
\hline Distribution & 9,290 & 0 & 9,290 & 0 & 9,902 & 0 & 10,562 & 0 \\
\hline Transportation & 1,366 & 1,852 & 1,350 & 1,869 & 1,228 & 1,230 & 1,320 & 1,275 \\
\hline Finance & 6,505 & 901 & 6,572 & 920 & 6,105 & 945 & 6,452 & 980 \\
\hline Social services & 4,475 & 20,531 & 4,649 & 20,674 & 4,335 & 21,177 & 5,121 & 21,952 \\
\hline Total & 63,598 & 28,554 & 64,817 & 28,754 & 66,267 & 29,091 & 72,216 & 30,156 \\
\hline \multicolumn{9}{|l|}{ Memorandum item: } \\
\hline Swazi mining migrant labor & 8,308 & & $\ldots$ & & $\ldots$ & & $\ldots$ & \\
\hline
\end{tabular}

Source: Ministry of Enterprise and Employment.

1/ Paid employment (including part-time) as of June of each year. 
Table 13. Swaziland: Summary of Central Government Operations, 2000/01-2004/05 ${ }^{1 /}$

\begin{tabular}{|c|c|c|c|c|c|}
\hline & $2000 / 01$ & $2001 / 02$ & $2002 / 03$ & $2003 / 04$ & $2004 / 05$ \\
\hline & \multicolumn{5}{|c|}{ (In millions of emalangeni) } \\
\hline Total revenue and grants & $2,825.3$ & $3,111.2$ & $3,425.9$ & $3,908.4$ & $4,841.8$ \\
\hline Tax revenue & $2,574.4$ & $2,785.2$ & $3,107.4$ & $3,660.5$ & $4,627.8$ \\
\hline SACU receipts & $1,406.7$ & $1,503.7$ & $1,618.6$ & $1,878.1$ & $2,772.8$ \\
\hline Non-SACU revenue & $1,167.7$ & $1,281.5$ & $1,488.8$ & $1,782.4$ & $1,855.0$ \\
\hline Nontax revenue & 138.8 & 199.1 & 155.4 & 110.2 & 98.0 \\
\hline Total expenditure and net lending & $2,968.3$ & $3,455.4$ & $4,018.2$ & $4,314.0$ & $5,554.0$ \\
\hline Current expenditure & $2,364.1$ & $2,542.4$ & $3,045.0$ & $3,437.2$ & $4,295.0$ \\
\hline Wages and salaries & $1,071.0$ & $1,155.3$ & $1,417.1$ & $1,668.9$ & $1,964.0$ \\
\hline Goods and services & 679.5 & 778.2 & 905.6 & $1,003.0$ & $1,421.0$ \\
\hline Interest payments & 63.1 & 102.1 & 167.3 & 173.8 & 168.0 \\
\hline Subsidies and transfers & 550.5 & 506.9 & 555.0 & 591.5 & 742.0 \\
\hline Capital expenditure & 604.4 & 867.1 & 935.5 & 817.8 & $1,259.0$ \\
\hline Net lending & -0.2 & 45.9 & 37.7 & 59.0 & 3.0 \\
\hline Primary balance & -79.9 & -242.1 & -425.0 & -231.8 & -544.2 \\
\hline Overall balance (including grants) & -143.0 & -344.2 & -592.3 & -405.6 & -712.2 \\
\hline Overall balance (excluding grants) & -255.1 & -471.0 & -755.4 & -543.3 & -828.2 \\
\hline Financing & 143.0 & 344.2 & 592.3 & 405.6 & 712.2 \\
\hline Foreign (net) & -23.5 & 112.6 & 218.4 & 77.0 & 255.0 \\
\hline Domestic (net) 2/ & 166.5 & 231.6 & 373.9 & 328.6 & 457.3 \\
\hline Government debt & $2,331.7$ & $2,785.7$ & $2,850.1$ & $2,955.3$ & $3,101.9$ \\
\hline Foreign & $2,261.7$ & $2,656.2$ & $2,499.7$ & $2,455.0$ & $2,461.6$ \\
\hline \multirow[t]{2}{*}{ Domestic } & 70.0 & 129.5 & 350.4 & 500.2 & 640.3 \\
\hline & \multicolumn{5}{|c|}{ (In percent of GDP, unless otherwise specified) } \\
\hline Total revenue and grants & 28.4 & 27.6 & 26.3 & 26.3 & 29.3 \\
\hline Tax revenue & 25.9 & 24.7 & 23.9 & 24.6 & 28.0 \\
\hline SACU receipts & 14.2 & 13.3 & 12.4 & 12.6 & 16.8 \\
\hline Non-SACU revenue & 11.7 & 11.4 & 11.4 & 12.0 & 11.2 \\
\hline Nontax revenue & 1.4 & 1.8 & 1.2 & 0.7 & 0.6 \\
\hline Grants & 1.1 & 1.1 & 1.3 & 0.9 & 0.7 \\
\hline Total expenditure and net lending & 29.9 & 30.6 & 30.8 & 29.0 & 33.6 \\
\hline Current expenditure & 23.8 & 22.6 & 23.4 & 23.1 & 26.0 \\
\hline \multicolumn{6}{|l|}{ Of which: } \\
\hline Wages and salaries & 10.8 & 10.2 & 10.9 & 11.2 & 11.9 \\
\hline Goods and services & 6.8 & 6.9 & 7.0 & 6.7 & 8.6 \\
\hline Interest payments & 0.6 & 0.9 & 1.3 & 1.2 & 1.0 \\
\hline Subsidies and transfers & 5.5 & 4.5 & 4.3 & 4.0 & 4.5 \\
\hline Capital expenditure & 6.1 & 7.7 & 7.2 & 5.5 & 7.6 \\
\hline Primary balance & -0.8 & -2.1 & -3.3 & -1.6 & -3.3 \\
\hline Overall balance (including grants) & -1.4 & -3.1 & -4.5 & -2.7 & -4.3 \\
\hline Overall balance (excluding grants) & -2.6 & -4.2 & -5.8 & -3.7 & -5.0 \\
\hline Financing & 1.4 & 3.1 & 4.5 & 2.7 & 4.3 \\
\hline Foreign (net) & -0.2 & 1.0 & 1.7 & 0.5 & 1.5 \\
\hline Domestic (net) & 1.7 & 2.1 & 2.9 & 2.2 & 2.8 \\
\hline Government debt & 23.5 & 24.7 & 21.9 & 19.9 & 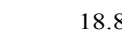 \\
\hline Foreign & 22.8 & 23.6 & 19.2 & 16.5 & 14.9 \\
\hline Domestic & 0.7 & 1.1 & 2.7 & 3.4 & 3.9 \\
\hline \multicolumn{6}{|l|}{ Memorandum items: } \\
\hline Payment arrears & $\ldots$ & $\ldots$ & $\ldots$ & 0.8 & 2.0 \\
\hline GDP at current prices (in millions of emalangeni) & 9,940 & 11,275 & 13,026 & 14,882 & 16,538 \\
\hline Wages and salaries (in percent of current expenditure) & 45.3 & 45.4 & 46.5 & 48.6 & 45.7 \\
\hline
\end{tabular}

Sources: Ministry of Finance; and Fund staff projections.

1/ The fiscal year runs from April 1 to March 31 .

2/ Including domestic payment arrears estimated at 2 percent of GDP for 2004/05. For 2005/06 onwards, including financing gaps.

\section{(C)International Monetary Fund. Not for Redistribution}


Table 14. Swaziland: Central Government Revenue and Grants, 2000/01-2004/05 1/

\begin{tabular}{|c|c|c|c|c|c|}
\hline & $2000 / 01$ & $2001 / 02$ & $2002 / 03$ & $2003 / 04$ & $2004 / 05$ \\
\hline & \multicolumn{5}{|c|}{ (In millions of emalangeni) } \\
\hline Tax revenue & $2,574.4$ & $2,785.2$ & $3,107.4$ & $3,660.5$ & $4,627.8$ \\
\hline Taxes on net income and profits & 678.0 & 725.0 & 827.5 & $1,105.4$ & $1,164.0$ \\
\hline Companies & 233.1 & 245.1 & 259.6 & 322.4 & 324.0 \\
\hline Individuals & 430.8 & 431.2 & 494.1 & 698.7 & 742.0 \\
\hline Nonresident dividends and interest & 14.2 & 48.8 & 73.8 & 84.3 & 98.0 \\
\hline Taxes on property & 5.4 & 5.5 & 6.0 & 9.0 & 12.0 \\
\hline Taxes on goods, services, and international trade & $1,886.0$ & $2,047.5$ & $2,269.2$ & $2,540.8$ & $3,445.8$ \\
\hline Southern African Customs Union (SACU) receipts & $1,406.7$ & $1,503.7$ & $1,618.6$ & $1,878.1$ & $2,772.8$ \\
\hline Sugar export levy & 15.5 & 28.5 & 21.8 & 12.1 & 22.0 \\
\hline Hotel and gaming taxes & 3.0 & 3.2 & 3.7 & 4.4 & 5.0 \\
\hline Licenses and other taxes & 89.1 & 104.4 & 96.6 & 98.5 & 97.0 \\
\hline Sales tax & 371.7 & 407.7 & 528.6 & 547.7 & 549.0 \\
\hline Road levy and oil tax & 0.0 & 0.0 & 0.0 & 0.0 & 0.0 \\
\hline Other taxes & 5.1 & 7.2 & 4.7 & 5.3 & 6.0 \\
\hline Nontax revenue & 138.8 & 199.1 & 157.1 & 104.9 & 98.0 \\
\hline Property income & 91.1 & 137.3 & 108.6 & 52.0 & 46.0 \\
\hline Fees, fines, and nonindustrial sales & 47.7 & 61.8 & 48.5 & 52.9 & 52.0 \\
\hline Total revenue & $2,713.2$ & $2,984.4$ & $3,264.5$ & $3,765.4$ & $4,725.8$ \\
\hline Grants & 112.1 & 126.9 & 163.0 & 137.7 & 116.0 \\
\hline \multirow[t]{2}{*}{ Total revenue and grants } & $2,825.3$ & $3,111.2$ & $3,427.6$ & $3,903.1$ & $4,841.8$ \\
\hline & \multicolumn{5}{|c|}{ (In percent of GDP) } \\
\hline Tax revenue & 25.9 & 24.7 & 23.9 & 24.6 & 28.0 \\
\hline Taxes on net income and profits & 6.8 & 6.4 & 6.4 & 7.4 & 7.0 \\
\hline \multicolumn{6}{|l|}{ Of which } \\
\hline Companies & 2.3 & 2.2 & 2.0 & 2.2 & 2.0 \\
\hline Individuals & 4.3 & 3.8 & 3.8 & 4.7 & 4.5 \\
\hline Taxes on property & 0.1 & 0.0 & 0.0 & 0.1 & 0.1 \\
\hline $\begin{array}{l}\text { Taxes on goods, services, and international trade } \\
\text { Of which }\end{array}$ & 19.0 & 18.2 & 17.4 & 17.1 & 20.8 \\
\hline SACU receipts & 14.2 & 13.3 & 12.4 & 12.6 & 16.8 \\
\hline Sales tax & 3.7 & 3.6 & 4.1 & 3.7 & 3.3 \\
\hline Other taxes & 0.1 & 0.1 & 0.0 & 0.0 & 0.0 \\
\hline Nontax revenue & 1.4 & 1.8 & 1.2 & 0.7 & 0.6 \\
\hline Grants & 1.1 & 1.1 & 1.3 & 0.9 & 0.7 \\
\hline \multirow[t]{2}{*}{ Total revenue and grants } & 28.4 & 27.6 & 26.3 & 26.2 & 29.3 \\
\hline & \multicolumn{5}{|c|}{ (In percent of total revenue and grants) } \\
\hline Tax revenue & 91.1 & 89.5 & 90.7 & 93.8 & 95.6 \\
\hline Taxes on net income and profits & 24.0 & 23.3 & 24.1 & 28.3 & 24.0 \\
\hline \multicolumn{6}{|l|}{ Of which } \\
\hline Companies & 8.2 & 7.9 & 7.6 & 8.3 & 6.7 \\
\hline Individuals & 15.2 & 13.9 & 14.4 & 17.9 & 15.3 \\
\hline Taxes on property & 0.2 & 0.2 & 0.2 & 0.2 & 0.2 \\
\hline Taxes on goods, services, and international trade & 66.8 & 65.8 & 66.2 & 65.1 & 71.2 \\
\hline Of which: SACU receipts & 49.8 & 48.3 & 47.2 & 48.1 & 57.3 \\
\hline Other taxes & 0.2 & 0.2 & 0.1 & 0.1 & 0.1 \\
\hline Nontax revenue & 4.9 & 6.4 & 4.6 & 2.7 & 2.0 \\
\hline Grants & 4.0 & 4.1 & 4.8 & 3.5 & 2.4 \\
\hline \multicolumn{6}{|l|}{ Memorandum item: } \\
\hline GDP at current market prices (in millions of emalangeni) & $9,939.7$ & $11,274.6$ & $13,025.6$ & $14,882.4$ & $16,537.5$ \\
\hline
\end{tabular}

Source: Ministry of Finance.

1/ Fiscal year runs from April 1 to March 31.

\section{CInternational Monetary Fund. Not for Redistribution}


Table 15. Swaziland: Functional Classification of Central Government Expenditure and Net Lending, 2000/01-2004/05 1/

\begin{tabular}{|c|c|c|c|c|c|}
\hline & $2000 / 01$ & $2001 / 02$ & $2002 / 03$ & $2003 / 04$ & $2004 / 05$ \\
\hline & \multicolumn{5}{|c|}{ (In millions of emalangeni) } \\
\hline Current expenditure & $2,364.1$ & $2,542.4$ & $3,045.0$ & $3,437.2$ & $4,294.9$ \\
\hline Wages and salaries & $1,071.0$ & $1,155.3$ & $1,417.1$ & $1,668.9$ & $1,964.0$ \\
\hline Of which: Health & & 92.0 & 101.0 & 131.0 & 165.2 \\
\hline Education & & 409.0 & 488.0 & 575.0 & 715.4 \\
\hline Other purchases of goods and services & 679.5 & 778.2 & 905.6 & $1,003.0$ & $1,421.0$ \\
\hline Of which: Health & & 79.0 & 93.0 & 119.0 & 142.8 \\
\hline Education & & 49.0 & 39.0 & 64.0 & 69.1 \\
\hline Interest payments & 63.1 & 102.1 & 167.3 & 173.8 & 168.0 \\
\hline Domestic & 5.6 & 5.6 & 0.0 & 37.0 & 49.0 \\
\hline Foreign & 57.5 & 96.4 & 167.3 & 136.8 & 119.0 \\
\hline Subsidies and other current transfers & 550.5 & 506.9 & 555.0 & 591.5 & 742.0 \\
\hline Of which: subsidies to NFPEs 2/ & 147.5 & 276.2 & 246.5 & 200.7 & 213.2 \\
\hline Capital expenditure & 604.4 & 867.1 & 935.5 & 817.8 & $1,259.0$ \\
\hline Of which: Health & 33.0 & 26.0 & 22.0 & 60.0 & 82.6 \\
\hline Education & 27.0 & 44.0 & 48.0 & 13.0 & 27.9 \\
\hline Agriculture & 60.0 & 63.0 & 31.0 & 48.0 & 104.5 \\
\hline Transport and communications & 179.0 & 348.0 & 427.0 & 125.0 & 347.6 \\
\hline Other & 338.4 & 386.1 & 407.5 & 571.8 & 696.4 \\
\hline Net lending & -0.2 & 45.9 & 37.7 & 59.0 & 3.0 \\
\hline Gross lending & 19.2 & 52.1 & 44.3 & 68.3 & 10.0 \\
\hline Repayments & -19.4 & -6.2 & -6.5 & -9.3 & -7.0 \\
\hline \multirow[t]{2}{*}{ Total expenditure and net lending } & $2,968.3$ & $3,455.4$ & $4,018.2$ & $4,314.0$ & $5,556.9$ \\
\hline & \multicolumn{5}{|c|}{ (In percent of GDP) } \\
\hline Current expenditure & 23.8 & 22.6 & 23.4 & 23.1 & 26.0 \\
\hline Capital expenditure & 6.1 & 7.7 & 7.2 & 5.5 & 7.6 \\
\hline Net lending & 0.0 & 0.4 & 0.3 & 0.4 & 0.0 \\
\hline \multirow[t]{2}{*}{ Total expenditure and net lending } & 29.9 & 30.6 & 30.8 & 29.0 & 33.6 \\
\hline & \multicolumn{5}{|c|}{ (In percent of total expenditure and net lending, unless otherwise indicated) } \\
\hline Current expenditure & 79.6 & 73.6 & 75.8 & 79.7 & 77.3 \\
\hline Wages and salaries & 36.1 & 33.4 & 35.3 & 38.7 & 35.3 \\
\hline Other purchases of goods and services & 22.9 & 22.5 & 22.5 & 23.2 & 25.6 \\
\hline Interest payments & 2.1 & 3.0 & 4.2 & 4.0 & 3.0 \\
\hline Subsidies and other current transfers & 18.5 & 14.7 & 13.8 & 13.7 & 13.4 \\
\hline Capital expenditure & 20.4 & 25.1 & 23.3 & 19.0 & 22.7 \\
\hline Net lending & 0.0 & 1.3 & 0.9 & 1.4 & 0.1 \\
\hline \multicolumn{6}{|l|}{ Memorandum item: } \\
\hline GDP at current market prices (in millions of emalangeni) & $9,939.7$ & $11,274.6$ & $13,025.6$ & $14,882.4$ & $16,537.5$ \\
\hline
\end{tabular}

Source: Ministry of Finance.

1/ Fiscal year runs from April 1 to March 31.

2/ NFPEs (nonfinancial public enterprises). 
Table 16. Swaziland: Economic Classification of Government Current Expenditure, 2000/01-2004/05 ${ }^{\text {1/ }}$

\begin{tabular}{|c|c|c|c|c|c|}
\hline & $2000 / 01$ & $2001 / 02$ & $2002 / 03$ & $2003 / 04$ & $2004 / 05$ \\
\hline & \multicolumn{5}{|c|}{ (In millions of emalangeni) } \\
\hline General public service & $1,019.0$ & $1,022.0$ & $1,213.0$ & $1,183.0$ & $1,448.4$ \\
\hline General administration & 756.0 & 739.0 & 907.0 & 495.0 & 962.9 \\
\hline Public safety and defense & 263.0 & 283.0 & 306.0 & 688.0 & 485.4 \\
\hline Defense & 173.0 & 168.0 & 202.0 & 255.0 & 282.8 \\
\hline Social services & 814.0 & 902.0 & $1,047.6$ & $1,358.0$ & $1,653.0$ \\
\hline Education & 552.0 & 607.0 & 711.0 & 911.0 & $1,130.3$ \\
\hline Health & 197.0 & 210.0 & 239.6 & 291.0 & 348.0 \\
\hline Other & 65.0 & 85.0 & 97.0 & 156.0 & 174.7 \\
\hline Economic services & 296.1 & 348.4 & 415.4 & 507.2 & 742.7 \\
\hline Agriculture & 87.5 & 104.0 & 120.8 & 147.0 & 203.0 \\
\hline Manufacturing, mining, and construction & 45.0 & 45.0 & 46.8 & 58.0 & 69.0 \\
\hline Water and sewerage & 20.0 & 22.0 & 24.0 & 31.0 & 36.0 \\
\hline Transport and communications & 132.0 & 160.0 & 231.0 & 187.0 & 231.0 \\
\hline Other & 11.6 & 17.4 & -7.2 & 84.2 & 203.7 \\
\hline Interest payments & 62.0 & 102.0 & 167.0 & 134.0 & 168.0 \\
\hline \multirow[t]{2}{*}{ Total } & $2,364.1$ & $2,542.4$ & $3,045.0$ & $3,437.2$ & $4,294.9$ \\
\hline & \multicolumn{5}{|c|}{ (In percent of GDP) } \\
\hline General public service & 10.3 & 9.1 & 9.3 & 7.9 & 8.8 \\
\hline General administration & 7.6 & 6.6 & 7.0 & 3.3 & 5.8 \\
\hline Public safety and defense & 2.6 & 2.5 & 2.3 & 4.6 & 2.9 \\
\hline Defense & 1.7 & 1.5 & 1.6 & 1.7 & 1.7 \\
\hline Social services & 8.2 & 8.0 & 8.0 & 9.1 & 10.0 \\
\hline Education & 5.6 & 5.4 & 5.5 & 6.1 & 6.8 \\
\hline Health & 2.0 & 1.9 & 1.8 & 2.0 & 2.1 \\
\hline Other & 0.7 & 0.8 & 0.7 & 1.0 & 1.1 \\
\hline Economic services & 3.0 & 3.1 & 3.2 & 3.4 & 4.5 \\
\hline \multirow[t]{2}{*}{ Interest payments } & 0.6 & 0.9 & 1.3 & 0.9 & 1.0 \\
\hline & \multicolumn{5}{|c|}{ (In percent of total current expenditure) } \\
\hline General public service & 43.1 & 40.2 & 39.8 & 34.4 & 33.7 \\
\hline General administration & 32.0 & 29.1 & 29.8 & 14.4 & 22.4 \\
\hline Public safety and defense & 11.1 & 11.1 & 10.0 & 20.0 & 11.3 \\
\hline Defense & 7.3 & 6.6 & 6.6 & 7.4 & 6.6 \\
\hline Social services & 34.4 & 35.5 & 34.4 & 39.5 & 38.5 \\
\hline Education & 23.3 & 23.9 & 23.4 & 26.5 & 26.3 \\
\hline Health & 8.3 & 8.3 & 7.9 & 8.5 & 8.1 \\
\hline Other & 2.7 & 3.3 & 3.2 & 4.5 & 4.1 \\
\hline Economic services & 12.5 & 13.7 & 13.6 & 14.8 & 17.3 \\
\hline Interest payments & 2.6 & 4.0 & 5.5 & 3.9 & 3.9 \\
\hline \multicolumn{6}{|l|}{ Memorandum item: } \\
\hline GDP at current market prices (in millions of emalangeni) & $9,939.7$ & $11,274.6$ & $13,025.6$ & $14,882.4$ & $16,537.5$ \\
\hline
\end{tabular}

Source: Ministry of Finance.

1/ Fiscal year runs from April 1 to March 31. 
Table 17. Swaziland: Capital Expenditure by Sector, 2000/01-2004/05 ${ }^{1 /}$ (In millions of emalangeni)

\begin{tabular}{|c|c|c|c|c|c|}
\hline & $2000 / 01$ & $2001 / 02$ & $2002 / 03$ & $2003 / 04$ & $2004 / 05$ \\
\hline General public services & 124.0 & 270.0 & 277.0 & 67.0 & 408.1 \\
\hline General administration & 103.0 & 244.0 & 225.0 & 28.0 & 365.6 \\
\hline Public safety and defense & 21.0 & 26.0 & 52.0 & 39.0 & 42.5 \\
\hline Social services & 133.0 & 124.0 & 137.0 & 282.0 & 360.0 \\
\hline Education & 27.0 & 44.0 & 48.0 & 13.0 & 28.0 \\
\hline Health & 33.0 & 26.0 & 22.0 & 60.0 & 83.0 \\
\hline Housing & 58.0 & 43.0 & 62.0 & 193.0 & 247.0 \\
\hline Recreation and culture & 1.0 & 0.0 & 0.0 & 7.0 & 1.1 \\
\hline Other & 14.0 & 11.0 & 5.0 & 9.0 & 0.9 \\
\hline Economic services & 348.0 & 473.0 & 521.5 & 468.8 & 490.9 \\
\hline Agriculture & 60.0 & 63.0 & 31.0 & 48.0 & 105.0 \\
\hline Industry and mining & 81.0 & 6.0 & 4.0 & 4.0 & 23.0 \\
\hline Water and sewage & 26.0 & 1.0 & 12.0 & 13.0 & 9.0 \\
\hline Transport and communications & 179.0 & 348.0 & 427.0 & 125.0 & 348.0 \\
\hline Other & 2.0 & 55.0 & 47.5 & 278.8 & 5.9 \\
\hline Total capital expenditure & 605.0 & 867.0 & 935.5 & 817.8 & $1,259.0$ \\
\hline
\end{tabular}

Source: Ministry of Finance.

1/ Fiscal year runs from April 1 to March 31. 
Table 18. Swaziland: Government Transfer Payments, 2000/01-2003/04 1/ (In millions of emalangeni, unless otherwise indicated)

\begin{tabular}{lrrrr}
\hline & $2000 / 01$ & $2001 / 02$ & $2002 / 03$ & $2003 / 04$ \\
& & & & \\
\hline & 69.5 & 66.2 & 68.5 & 109.0 \\
Grants to students & 43.0 & 37.1 & 37.2 & 42.1 \\
Grants to mission hospital & 2.1 & 2.2 & 0.8 & 2.2 \\
Public assistance & 22.1 & 29.0 & 30.1 & 38.7 \\
Grants to town councils & 6.7 & 5.1 & 5.1 & 5.0 \\
Grants to youth and sports & 20.3 & 23.8 & 24.4 & 29.4 \\
External transfers 2/ & 7.0 & 53.5 & 57.4 & 54.4 \\
Swaziland National Treasury & 23.2 & 8.9 & 5.5 & 5.5 \\
Cattle-dipping chemicals & 6.5 & 7.8 & 7.8 & 9.2 \\
Swaziland Komati Project Enterprise & 52.4 & 99.6 & 140.1 & 125.9 \\
Other & & & & \\
& 252.8 & 333.2 & 376.9 & 421.4 \\
Total & & & & \\
Memorandum item: & & & & \\
Transfers (in percent of GDP) & 2.5 & 3.0 & 2.9 & 2.8 \\
Nominal GDP (in millions of emalangeni) & 9,940 & 11,275 & 13,026 & 14,882 \\
& & & & \\
\hline
\end{tabular}

Source: Ministry of Finance.

1/ Fiscal year runs from April 1 to March 31.

2/ Subscriptions to international organizations. 
Table 19. Swaziland: Flow of Funds From the Government to Public Enterprises 2000/01-2004/2005 ${ }^{1 /}$ (In millions of emalangeni, unless otherwise indicated)

\begin{tabular}{|c|c|c|c|c|c|}
\hline & $2000 / 01$ & $2001 / 02$ & $2002 / 03$ & $2003 / 04$ & $2004 / 05$ \\
\hline Total subsidies to public enterprises & 266.1 & 396.2 & 306.5 & 200.7 & 213.2 \\
\hline Operating/Transport subsidy & 125.7 & 128.6 & 175.8 & 169.5 & 172.5 \\
\hline Capital subsidy (flow) & 140.4 & 267.6 & 130.7 & 31.2 & 40.7 \\
\hline Total subsidies to public enterprises & 266.1 & 396.2 & 306.5 & 200.7 & 213.2 \\
\hline Swaziland Development \& Savings Bank & 120.0 & 120.0 & 60.0 & 0.0 & 0.0 \\
\hline Subsidies to nonfinancial public enterprises & 146.1 & 276.2 & 246.5 & 200.7 & 213.2 \\
\hline Joint Venture Capital Fund & 0.0 & 80.0 & 0.0 & 0.0 & 0.0 \\
\hline Swaziland Water Services Corporation & 1.1 & 2.6 & 9.9 & 0.0 & 0.0 \\
\hline Swaziland Investment Promotion Authority & 3.7 & 3.9 & 4.6 & 3.9 & 4.7 \\
\hline Swaziland Television Authority & 4.3 & 18.4 & 10.1 & 10.1 & 10.1 \\
\hline Sebenta National Institute & 2.4 & 2.8 & 3.0 & 2.7 & 2.7 \\
\hline Swaziland National Trust Commission & 7.0 & 10.2 & 7.2 & 7.3 & 8.3 \\
\hline University of Swaziland & 63.9 & 64.6 & 92.4 & 109.3 & 109.3 \\
\hline Motor Vehicle Accident Fund & 34.0 & 28.0 & 28.0 & 0.0 & 0.0 \\
\hline Swaziland Komati Project Enterprise & 25.6 & 37.3 & 57.5 & 38.6 & 38.6 \\
\hline Commission for Mediation etc. & 0.0 & 0.0 & 2.2 & 2.6 & 5.4 \\
\hline National Emergency Response Committee & 0.0 & 0.0 & 20.0 & 20.0 & 20.0 \\
\hline Other & 4.1 & 28.4 & 11.6 & 6.2 & 14.1 \\
\hline \multicolumn{6}{|l|}{ Memorandum item: } \\
\hline Total Subsidies (in percent of GDP) & 2.7 & 3.5 & 2.4 & 1.3 & 1.3 \\
\hline Nominal GDP (in millions of emalangeni) & 9,940 & 11,275 & 13,026 & 14,882 & 16,538 \\
\hline
\end{tabular}

Source: Ministry of Finance.

1/ Fiscal year runs from April 1 to March 31. 
Table 20. Swaziland: Outstanding Domestic Government Debt by Type of Instrument, 2000-2004 (In millions of emalangeni; end of period)

\begin{tabular}{|c|c|c|c|c|c|}
\hline & 2000 & 2001 & 2002 & 2003 & 2004 \\
\hline Government bonds and stocks & 30.0 & 30.0 & 260.0 & 260.0 & 180.0 \\
\hline Central Bank of Swaziland & 0.0 & 0.0 & 0.0 & 0.0 & 0.0 \\
\hline Banks & 20.3 & 20.3 & 176.7 & 176.7 & 146.3 \\
\hline Other financial institutions & 1.0 & 1.0 & 53.9 & 53.9 & 14.5 \\
\hline Other & 8.7 & 8.7 & 29.4 & 29.4 & 19.2 \\
\hline Treasury bills $1 /$ & 40.0 & 40.0 & 40.0 & 217.7 & 374.0 \\
\hline Central Bank of Swaziland & 0.0 & 0.0 & 0.0 & 40.0 & 73.0 \\
\hline Banks & 40.0 & 40.0 & 40.0 & 160.0 & 280.0 \\
\hline Other & 0.0 & 0.0 & 0.0 & 17.7 & 21.0 \\
\hline Total domestic government debt & 70.0 & 70.0 & 300.0 & 477.7 & 554.0 \\
\hline \multicolumn{6}{|l|}{ By original maturity } \\
\hline Less than one year & 40.0 & 40.0 & 40.0 & 217.7 & 374.0 \\
\hline From one to five years & 0.0 & 30.0 & 210.0 & 210.0 & 130.0 \\
\hline From five to ten years & 30.0 & 0.0 & 50.0 & 50.0 & 50.0 \\
\hline Over ten years & 0.0 & 0.0 & 0.0 & 0.0 & 0.0 \\
\hline
\end{tabular}

Source: Central Bank of Swaziland.

$1 /$ The maturity of treasury bills is three months. 
Table 21. Swaziland: Monetary Survey, 2001-2005 ${ }^{1 /}$

\begin{tabular}{|c|c|c|c|c|c|}
\hline & 2001 & 2002 & 2003 & 2004 & $\begin{array}{c}2005 \\
\text { Sept } \\
\end{array}$ \\
\hline & \multicolumn{5}{|c|}{ (In millions of Emalangeni) } \\
\hline \multicolumn{6}{|l|}{ Monetary authorities } \\
\hline Net foreign assets & $2,770.5$ & $1,873.5$ & $1,404.7$ & $1,450.2$ & $1,461.0$ \\
\hline Central Bank of Swaziland (CBS) & $2,388.9$ & $1,863.3$ & $1,378.2$ & $1,243.3$ & $1,452.0$ \\
\hline Of which: Capital Investment Fund (CIF), managed by CBS. & $1,537.4$ & $1,219.7$ & 712.9 & 666.7 & 569.0 \\
\hline Government & 381.7 & 10.2 & 26.5 & 206.9 & 9.0 \\
\hline Net domestic assets & $-2,517.4$ & $-1,543.7$ & -974.8 & -973.2 & -973.0 \\
\hline Central government (net) & $-2,112.4$ & $-1,299.7$ & $-1,030.8$ & -933.2 & -864.0 \\
\hline CBS claims on government & 0.0 & 57.3 & 82.6 & 251.8 & 187.0 \\
\hline Government deposits with CBS & $-2,112.4$ & $-1,357.0$ & $-1,113.4$ & $-1,185.0$ & $-1,051.0$ \\
\hline Domestic & -193.3 & -127.1 & -374.0 & -311.3 & -473.0 \\
\hline Foreign $2 /$ & $-1,919.0$ & $-1,229.9$ & -739.4 & -873.6 & -578.0 \\
\hline Private sector & 15.0 & 13.0 & 12.0 & 10.0 & 8.0 \\
\hline Commercial banks (net) & 0.0 & 0.0 & 0.0 & 0.0 & 0.0 \\
\hline Other items (net) & -420.0 & -257.0 & 44.0 & -50.0 & -117.0 \\
\hline Reserve money & 254.1 & 329.4 & 430.3 & 477.1 & 488.0 \\
\hline \multicolumn{6}{|l|}{ Commercial banks } \\
\hline Net foreign assets & 865.8 & 715.0 & 469.4 & 490.4 & 304.0 \\
\hline Reserves & 110.0 & 176.0 & 194.0 & 211.0 & 211.0 \\
\hline Domestic credit & $1,443.3$ & $1,985.3$ & $2,614.1$ & $3,344.2$ & $3,836.0$ \\
\hline Central government (net) & 59.8 & 215.2 & 286.4 & 331.0 & 326.0 \\
\hline Claims on Government & 60.0 & 215.4 & 286.7 & 332.5 & 345.0 \\
\hline Government deposits & 0.2 & 0.2 & 0.3 & 1.5 & 19.0 \\
\hline Private sector & $1,383.6$ & $1,770.1$ & $2,327.7$ & $3,013.2$ & $3,510.0$ \\
\hline Other items (net) & -332.0 & -519.0 & -613.0 & $-1,100.0$ & $-1,275.0$ \\
\hline Private sector deposits & $2,086.5$ & $2,357.0$ & $2,663.7$ & $2,945.9$ & $3,076.0$ \\
\hline \multicolumn{6}{|l|}{ Monetary survey } \\
\hline Net foreign assets & $3,636.4$ & $2,588.5$ & $1,874.1$ & $1,940.6$ & $1,765.0$ \\
\hline Domestic credit & -654.0 & 698.7 & $1,595.3$ & $2,421.0$ & $2,980.0$ \\
\hline Central government (net) & $-2,052.6$ & $-1,084.5$ & -744.4 & -602.2 & -538.0 \\
\hline Private sector & $1,398.6$ & $1,783.1$ & $2,339.7$ & $3,023.2$ & $3,518.0$ \\
\hline Other items (net) & -681.4 & -684.7 & -500.4 & $-1,083.6$ & $-1,308.0$ \\
\hline Broad money & $2,300.9$ & $2,602.4$ & $2,969.0$ & $3,278.0$ & $3,437.0$ \\
\hline Currency in circulation 3/ & 134.9 & 155.4 & 213.5 & 235.8 & 271.0 \\
\hline \multirow[t]{2}{*}{ Deposits } & $2,166.0$ & $2,447.0$ & $2,755.5$ & $3,042.2$ & $3,166.0$ \\
\hline & \multicolumn{5}{|c|}{ (Annual change in percent of beginning-of-period broad money) ${ }^{4 /}$} \\
\hline Broad money & 10.7 & 13.1 & 14.1 & 10.4 & 4.9 \\
\hline Net foreign assets & 42.1 & -45.5 & -27.5 & 2.2 & -5.4 \\
\hline Domestic credit & -9.9 & 58.8 & 34.5 & 27.8 & 17.1 \\
\hline Central government (net) & -16.7 & 42.1 & 13.1 & 4.8 & 2.0 \\
\hline Private sector & 6.8 & 16.7 & 21.4 & 23.0 & 15.1 \\
\hline Other items (net) & -21.5 & -0.1 & 7.1 & -19.6 & -6.8 \\
\hline \multicolumn{6}{|l|}{ Memorandum items: } \\
\hline Currency/broad money (percent) & 5.9 & 6.0 & 7.2 & 7.2 & 7.9 \\
\hline Reserve money/deposits (percent) & 11.7 & 13.5 & 15.6 & 15.7 & 15.4 \\
\hline Money multiplier (broad money/reserve money) & 9.1 & 7.9 & 6.9 & 6.9 & 7.0 \\
\hline
\end{tabular}

Sources: Central Bank of Swaziland (CBS); and Fund staff estimates.

1/ End-of-year data.

2/ Counterpart of government external assets in rand and in CIF.

$3 /$ Excludes rand in circulation.

4/ For September 2005, change from Dec. 2004. 
Table 22. Swaziland: Assets and Liabilities of the Central Bank of Swaziland, 2000 - Nov. 2005

(In millions of emalangeni, end of period)

\begin{tabular}{|c|c|c|c|c|c|c|c|}
\hline & 2000 & 2001 & 2002 & 2003 & 2004 & $\begin{array}{l}2005 \\
\text { Sept. }\end{array}$ & $\begin{array}{l}2005 \\
\text { Nov. }\end{array}$ \\
\hline Foreign assets & 2,562 & 3,242 & 2,472 & 1,758 & 1,474 & 1,487 & 1,934 \\
\hline CBS managed $1 /$ & 1,945 & 2,861 & 2,462 & 1,732 & 1,267 & 1,478 & 1,725 \\
\hline Government & 617 & 382 & 10 & 27 & 207 & 9 & 209 \\
\hline Claims on government & 0 & 0 & 57 & 83 & 252 & 187 & 242 \\
\hline Claims on private sector & 16 & 15 & 13 & 12 & 10 & 8 & 8 \\
\hline Claims on commercial banks & 42 & 42 & 0 & 0 & 0 & 0 & 0 \\
\hline Assets $=$ liabilities & 2,621 & 3,300 & 2,543 & 1,853 & 1,736 & 1,682 & 2,184 \\
\hline Foreign liabilities & 346 & 473 & 371 & 355 & 25 & 27 & 21 \\
\hline Government deposits 2/ & 1,765 & 1,731 & 1,347 & 1,087 & 978 & 1,041 & 1,549 \\
\hline Of which: Capital Investment Fund & 941 & 1,537 & 1,220 & 713 & 667 & 569 & 586 \\
\hline Private sector deposits & 68 & 80 & 90 & 92 & 96 & 98 & 101 \\
\hline Commercial banks & 10 & 0 & 0 & 0 & 0 & 0 & 44 \\
\hline Reserve money & 277 & 254 & 329 & 430 & 477 & 488 & 493 \\
\hline Currency in circulation $3 /$ & 214 & 202 & 227 & 286 & 302 & 318 & 329 \\
\hline Commercial bank deposits & 62 & 52 & 102 & 145 & 175 & 170 & 164 \\
\hline Other items (net) $4 /$ & 155 & 762 & 406 & -111 & 159 & 27 & -24 \\
\hline
\end{tabular}

Source: Central Bank of Swaziland (CBS).

1/ Includes Capital Investment Fund.

2/ Includes counterpart of government foreign assets.

3/ Excludes rand in circulation.

4/ Includes balances held with other banks in Swaziland, bills payable, unclassified assets, and unclassified liabilities. 
Table 23. Swaziland: Assets and Liabilities of Commercial Banks, 2000 - Nov. 2005 1/

(In millions of emalangeni, end of period)

\begin{tabular}{|c|c|c|c|c|c|c|c|}
\hline & 2000 & 2001 & 2002 & 2003 & 2004 & $\begin{array}{l}2005 \\
\text { Sept. }\end{array}$ & $\begin{array}{l}2005 \\
\text { Nov. }\end{array}$ \\
\hline Foreign assets & 654 & 892 & 791 & 521 & 531 & 399 & 418 \\
\hline Claims on government & 59 & 60 & 215 & 287 & 332 & 345 & 340 \\
\hline Claims on private sector & 1,308 & 1,384 & 1,770 & 2,328 & 3,013 & 3,510 & 3,533 \\
\hline Reserves & 138 & 132 & 209 & 236 & 256 & 211 & 230 \\
\hline Balances with CBS & 62 & 43 & 104 & 121 & 146 & 164 & 109 \\
\hline Cash in tills $2 /$ & 66 & 67 & 72 & 72 & 66 & 47 & 77 \\
\hline CBS bills & 10 & 80 & 25 & 0 & 0 & 0 & 44 \\
\hline Other items (net) $3 /$ & -32 & -62 & -174 & -168 & -103 & -176 & -220 \\
\hline Assets $=$ liabilities & 2,128 & 2,406 & 2,812 & 3,204 & 4,030 & 4,289 & 4,302 \\
\hline Foreign liabilities & 108 & 26 & 76 & 52 & 41 & 95 & 65 \\
\hline Government deposits & 0 & 0 & 0 & 0 & 2 & 19 & 24 \\
\hline Private sector deposits & 1,862 & 2,086 & 2,357 & 2,664 & 2,946 & 3,076 & 3,119 \\
\hline Demand & 513 & 619 & 670 & 834 & 844 & 965 & 956 \\
\hline Time & 961 & 1,086 & 1,255 & 1,353 & 1,517 & 1,487 & 1,530 \\
\hline Savings & 388 & 381 & 431 & 476 & 585 & 623 & 633 \\
\hline Balances due to CBS & 0 & 0 & 0 & 0 & 34 & 0 & 0 \\
\hline Capital accounts & 158 & 293 & 378 & 488 & 1,007 & 1,100 & 1,094 \\
\hline
\end{tabular}

Source: Central Bank of Swaziland (CBS).

1/ Includes the Swaziland Development and Savings Bank.

2/ Excludes rand in circulation.

3/ Includes balances held with other banks in Swaziland, bills payable, unclassified assets, and unclassified liabilities. 
Table 24. Swaziland: Commercial Banks' Deposits by Sector, 2000 - Sept. 2005 1/

(In millions of emalangeni, end of period)

\begin{tabular}{lrrrrrr}
\hline & 2000 & 2001 & 2002 & 2003 & 2004 & 2005 \\
& & & & & & Sept. \\
& 512 & 619 & 670 & 834 & 845 & 977 \\
Demand deposits & 2 & 3 & 3 & 3 & 1 & 12 \\
Central government & 42 & 40 & 50 & 49 & 23 & 58 \\
Other public bodies 2/ & 162 & 179 & 201 & 264 & 201 & 189 \\
Personal & 306 & 397 & 416 & 517 & 620 & 717 \\
Business & & & & & & \\
& 368 & 382 & 432 & 477 & 586 & 624 \\
Savings deposits & 0 & 0 & 0 & 0 & 0 & 0 \\
Central government & 2 & 2 & 2 & 2 & 0 & 5 \\
Other public bodies 2/ & 345 & 355 & 403 & 436 & 492 & 525 \\
Personal & 21 & 24 & 27 & 38 & 94 & 94 \\
Business & & & & & & \\
& 982 & 1,086 & 1,255 & 1,353 & 1517 & 1494 \\
Time deposits & 0 & 0 & 0 & 0 & 0 & 7 \\
Central government & 252 & 270 & 245 & 291 & 106 & 384 \\
Other public bodies 2/ & 151 & 160 & 233 & 264 & 230 & 159 \\
Personal & 579 & 656 & 777 & 798 & 1182 & 945 \\
Business & & & & & & \\
& 1,861 & 2,086 & 2,357 & 2,663 & 2948 & 3095 \\
Total deposits & 2 & 0 & 0 & 0 & 2 & 19 \\
Central government & 295 & 312 & 297 & 342 & 129 & 447 \\
Other public bodies 2/ & 657 & 695 & 837 & 964 & 922 & 873 \\
Personal & 906 & 1,076 & 1,220 & 1,353 & 1,896 & 1,756 \\
Business & & & & & & \\
& & & & & &
\end{tabular}

Source: Central Bank of Swaziland (CBS).

1/ Excluding bank checks outstanding.

2/ Comprises local government, city councils, and statutory corporations. 
Table 25. Swaziland: Commercial Banks' Liquidity Position, 2000 - Sept. 2005

(In millions of emalangeni, end of period)

\begin{tabular}{|c|c|c|c|c|c|c|}
\hline & 2000 & 2001 & 2002 & 2003 & 2004 & $\begin{array}{l}2005 \\
\text { Sept. }\end{array}$ \\
\hline Total liquid assets $1 /$ & 350 & 390 & 495 & 508 & 561 & 565 \\
\hline Of which: balance with CBS & 62 & 43 & 104 & 121 & 146 & 164 \\
\hline Required liquid assets & 307 & 347 & 383 & 371 & 415 & 427 \\
\hline Domestic liquidity surplus & 43 & 43 & 112 & 137 & 146 & 138 \\
\hline Net due from foreign banks & 516 & 835 & 695 & 397 & 394 & 223 \\
\hline Total liquidity surplus & 559 & 878 & 807 & 533 & 540 & 361 \\
\hline \multicolumn{7}{|l|}{ Memorandum items: } \\
\hline \multicolumn{7}{|l|}{ Liquidity ratios (in percent) } \\
\hline Required liquidity to deposits & 16 & 14 & 14 & 13 & 13 & 13 \\
\hline Actual liquidity to deposits & 19 & 16 & 18 & 17 & 17 & 17 \\
\hline
\end{tabular}

Source: Central Bank of Swaziland (CBS).

1/ Comprises cash in tills, treasury bills and eligible stock issued by the Swazi government, balances with the CBS and other banks, and other approved liquid assets. Also including rand notes and coins held by banks. 
Table 26. Swaziland: Commercial Bank Loans and Advances by Category of Borrower, 2000 - Sept. 2005 (In millions of emalangeni, end of period)

\begin{tabular}{|c|c|c|c|c|c|c|}
\hline & 2000 & 2001 & 2002 & 2003 & 2004 & $\begin{array}{c}2005 \\
\text { Sept }\end{array}$ \\
\hline Industry & 925 & 969 & 1,224 & 1,578 & 1877 & 2327 \\
\hline Agriculture and forestry & 155 & 224 & 304 & 365 & 403 & 527 \\
\hline Mining and quarrying & 4 & 4 & 6 & 7 & 0 & 0 \\
\hline Manufacturing & 398 & 349 & 311 & 486 & 428 & 498 \\
\hline Construction & 40 & 59 & 83 & 118 & 136 & 165 \\
\hline Distribution and tourism & 140 & 136 & 163 & 178 & 307 & 378 \\
\hline Transport and communications & 40 & 39 & 82 & 115 & 196 & 188 \\
\hline Social and personal services & 70 & 80 & 141 & 138 & 137 & 145 \\
\hline Other & 78 & 76 & 133 & 171 & 269 & 426 \\
\hline Personal loans 1/ & 215 & 185 & 309 & 530 & 874 & 973 \\
\hline Statutory bodies & 28 & 34 & 50 & 38 & 70 & 99 \\
\hline Other 2/ & 51 & 55 & 70 & 91 & 10 & 14 \\
\hline Total & 1,219 & 1,243 & 1,653 & 2,237 & 2,830 & 3,413 \\
\hline
\end{tabular}

Source: Central Bank of Swaziland (CBS).

1/ Includes small amounts lent to nonresidents.

2/ Local authorities and private financial institutions. 
Table 27. Interest Rates in Swaziland and South Africa, Dec. 2001 - Nov. 2005

(In percent)

\begin{tabular}{|c|c|c|c|c|c|c|c|c|c|c|}
\hline & 2001 & 2002 & 2003 & 2004 & 2004 & 2004 & 2004 & 2005 & 2005 & 2005 \\
\hline & Dec. & Dec. & Dec. & Mar. & Jun. & Sep. & Dec. & Mar. & Jun. & Nov. \\
\hline \multicolumn{11}{|l|}{ Discount rates } \\
\hline Central Bank of Swaziland & 9.5 & 13.5 & 8.0 & 8.0 & 8.0 & 7.5 & 7.5 & 7.5 & 7.0 & 7.0 \\
\hline South African Reserve Bank & 9.5 & 13.5 & 8.0 & 8.0 & 8.0 & 7.5 & 7.5 & 7.5 & 7.0 & 7.0 \\
\hline \multicolumn{11}{|l|}{ Treasury bills } \\
\hline Swaziland & 5.6 & 10.5 & 7.9 & 8.2 & 8.2 & 7.6 & 7.6 & 7.6 & 6.9 & 6.8 \\
\hline South Africa & 9.4 & 12.4 & 7.5 & 7.8 & 7.9 & 7.1 & 7.5 & 7.3 & 6.9 & 6.9 \\
\hline \multicolumn{11}{|l|}{ Prime lending rate } \\
\hline Swaziland & 12.5 & 16.5 & 11.5 & 11.5 & 11.5 & 11.0 & 11.0 & 11.0 & 10.5 & 10.5 \\
\hline South Africa & 13.0 & 17.0 & 11.5 & 11.5 & 11.5 & 11.0 & 11.0 & 11.0 & 10.5 & 10.5 \\
\hline \multicolumn{11}{|l|}{ Bank time deposits } \\
\hline Swaziland-31 days & 4.8 & 8.4 & 4.4 & 4.4 & 4.4 & 3.9 & 3.9 & 3.4 & 3.4 & 3.4 \\
\hline South Africa-31 days & 9.0 & 12.8 & 7.4 & 7.6 & 7.7 & 7.2 & 7.2 & 7.4 & 6.8 & 6.9 \\
\hline Swaziland-12 months & 6.3 & 9.5 & 4.2 & 4.2 & 4.2 & 4.1 & 4.1 & 4.1 & 3.5 & 3.5 \\
\hline South Africa-12 months & 10.2 & 12.9 & 7.9 & 8.6 & 8.9 & 7.5 & 7.3 & 7.7 & 7.1 & 7.3 \\
\hline \multicolumn{11}{|l|}{ Bank savings deposits, } \\
\hline Swaziland 1/ & $1.0-5.8$ & $6.8-9.8$ & $1.0-6.5$ & $1.0-6.5$ & $1.0-6.5$ & $0.0-6.5$ & $0.0-6.5$ & $0.0-5.0$ & $0.0-5.0$ & $0.0-5.0$ \\
\hline
\end{tabular}

Source: Central Bank of Swaziland (CBS).

1/ Simple average of end-of-period minimum and maximum rates across banks: rates on large-scale interbank deposits, as well as

other money rates, often diverge widely from the deposit rates offered to the general public in Swaziland. 
Table 28. Swaziland: Balance of Payments, 2000-2004

(In millions of U.S. dollars, unless otherwise specified)

\begin{tabular}{|c|c|c|c|c|c|}
\hline & 2000 & 2001 & 2002 & 2003 & 2004 \\
\hline Current account balance & -74.6 & -56.7 & 58.0 & 36.4 & 40.0 \\
\hline Trade balance & -136.2 & -81.8 & 90.7 & 42.8 & -32.0 \\
\hline Exports, f.o.b. & 905.6 & $1,043.3$ & $1,031.6$ & $1,484.9$ & $1,877.9$ \\
\hline Imports, f.o.b. & $-1,041.8$ & $-1,125.1$ & -940.8 & $-1,442.1$ & $-1,909.9$ \\
\hline Services (net) & -80.1 & -111.8 & -93.9 & -49.3 & -52.4 \\
\hline Exports of services & 228.2 & 113.8 & 102.2 & 156.5 & 482.3 \\
\hline Imports of services & -308.3 & -225.6 & -196.1 & -205.7 & -534.7 \\
\hline Goods and services balance & -216.3 & -193.6 & -3.2 & -6.5 & -84.4 \\
\hline Income (net) & 34.2 & 102.3 & -1.9 & -3.6 & 31.9 \\
\hline Income (credits) & 153.6 & 156.7 & 144.2 & 143.5 & 160.8 \\
\hline Income (debits) & -119.4 & -54.4 & -146.1 & -147.1 & -128.9 \\
\hline Of which: interest & -31.5 & -15.4 & -15.9 & -18.1 & -24.9 \\
\hline Transfers (net) & 107.5 & 34.5 & 63.1 & 46.4 & 92.4 \\
\hline Official sector (mainly SACU receipts) 1/ & 120.5 & 108.4 & 100.5 & 145.4 & 213.0 \\
\hline Private sector & -13.0 & -73.9 & -37.4 & -99.0 & -120.6 \\
\hline Capital and financial account balance & 17.1 & -67.3 & -52.6 & -28.5 & 28.7 \\
\hline Capital account balance & 0.1 & -0.2 & 0.5 & 0.0 & -0.6 \\
\hline Financial account balance (excluding reserve assets) & 17.0 & -67.1 & -53.1 & -28.5 & 29.2 \\
\hline Direct investment & 74.5 & 46.3 & 90.2 & -70.7 & 64.9 \\
\hline Portfolio investment & -0.6 & -7.9 & 1.9 & -2.2 & -11.0 \\
\hline Other investment & -56.9 & -105.5 & -145.2 & 44.4 & -24.7 \\
\hline Errors and omissions & 51.7 & 67.8 & -30.3 & -28.8 & -23.3 \\
\hline Overall balance & -5.8 & -56.3 & -24.9 & -20.9 & 45.3 \\
\hline \multicolumn{6}{|l|}{ Memorandum items: } \\
\hline Current account/GDP (in percent) & -5.4 & -4.5 & 4.9 & 1.9 & 1.6 \\
\hline Goods and services balance/GDP (in percent) & -15.6 & -15.4 & -0.3 & -0.3 & -3.3 \\
\hline Direct Investment/GDP (in percent) & 5.4 & 3.7 & 7.6 & -3.7 & 2.6 \\
\hline Net official reserves (end of period) & 292.9 & 228.5 & 216.8 & 211.5 & 257.6 \\
\hline In months of imports of goods and services & 2.6 & 2.0 & 2.3 & 1.5 & 1.3 \\
\hline Gross official reserves (end of period) & 338.5 & 267.4 & 259.5 & 264.8 & 261.8 \\
\hline In months of imports of goods and services & 3.0 & 2.4 & 2.7 & 1.9 & 1.3 \\
\hline External debt service (in percent of exports of goods and services) & 2.8 & 1.3 & 1.4 & 1.1 & 1.1 \\
\hline Total external debt & 358.1 & 288.4 & 350.8 & 412.9 & 571.0 \\
\hline Public & 273.5 & 233.9 & 295.5 & 352.2 & 501.0 \\
\hline Private & 84.6 & 54.6 & 55.3 & 60.7 & 70.0 \\
\hline Total external debt/GDP (in percent) & 25.8 & 22.9 & 29.4 & 21.7 & 22.6 \\
\hline GDP at current prices (at market exchange rates) & 1,390 & 1,261 & 1,194 & 1,907 & 2,521 \\
\hline Lilangeni per U.S. dollar (end of period) & 7.57 & 12.13 & 8.64 & 6.64 & 5.63 \\
\hline Lilangeni per U.S. dollar (period average) & 6.94 & 8.60 & 10.52 & 7.56 & 6.45 \\
\hline
\end{tabular}

Sources: Central Bank of Swaziland; and staff projections.

1/ SACU: Southern African Customs Union. 
Table 29. Swaziland: Balance of Payments, 2000-2004

(In millions of emalangeni, unless otherwise specified)

\begin{tabular}{|c|c|c|c|c|c|}
\hline & 2000 & 2001 & 2002 & 2003 & 2004 \\
\hline Current account balance & -517.6 & -488.2 & 610.3 & 275.3 & 257.7 \\
\hline Trade balance & -944.5 & -703.7 & 954.0 & 323.9 & -206.4 \\
\hline Exports, f.o.b. & $6,280.7$ & $8,975.3$ & $10,848.3$ & $11,232.6$ & $12,112.1$ \\
\hline Imports, f.o.b. & $-7,225.2$ & $-9,679.0$ & $-9,894.3$ & $-10,908.7$ & $-12,318.5$ \\
\hline Services (net) & -555.8 & -961.7 & -987.4 & -372.7 & -337.9 \\
\hline Exports of services & $1,582.5$ & 978.8 & $1,074.7$ & $1,183.5$ & $3,111.0$ \\
\hline Imports of services & $-2,138.3$ & $-1,940.5$ & $-2,062.1$ & $-1,556.2$ & $-3,448.9$ \\
\hline Goods and services balance & $-1,500.3$ & $-1,665.4$ & -33.4 & -48.8 & -544.3 \\
\hline Income (net) & 237.2 & 880.4 & -19.9 & -26.9 & 205.9 \\
\hline Income (credits) & $1,065.5$ & $1,348.5$ & $1,516.7$ & $1,085.7$ & $1,037.3$ \\
\hline Income (debits) & -828.3 & -468.1 & $-1,536.6$ & $-1,112.6$ & -831.4 \\
\hline Of which: interest & -218.8 & -132.7 & -167.0 & -137.1 & -160.7 \\
\hline Transfers (net) & 745.5 & 296.8 & 663.6 & 351.0 & 596.1 \\
\hline Official sector (mainly SACU receipts) 1/ & 835.7 & 932.6 & $1,056.9$ & $1,099.9$ & $1,373.9$ \\
\hline Private sector & -90.2 & -635.8 & -393.3 & -748.9 & -777.8 \\
\hline Capital and financial account balance & 118.5 & -579.3 & -553.4 & -215.5 & 184.8 \\
\hline Capital account balance & 0.6 & -1.7 & 4.9 & 0.0 & -3.7 \\
\hline Financial account balance (excluding reserve assets) & 117.8 & -577.6 & -558.3 & -215.5 & 188.5 \\
\hline Direct investment & 516.7 & 398.3 & 948.6 & -534.9 & 418.9 \\
\hline Portfolio investment & -4.2 & -68.0 & 20.0 & -16.4 & -70.8 \\
\hline Other investment & -394.7 & -908.0 & $-1,526.9$ & 335.9 & -159.6 \\
\hline Errors and omissions & 358.7 & 583.2 & -318.2 & -218.2 & -150.5 \\
\hline Overall balance & -40.4 & -484.3 & -261.3 & -158.4 & 292.0 \\
\hline \multicolumn{6}{|l|}{ Memorandum items: } \\
\hline GDP at current prices (at market exchange rates) & $9,637.6$ & $10,846.1$ & $12,560.0$ & $14,422.4$ & $16,262.4$ \\
\hline Lilangeni per U.S. dollar (end of period) & 7.57 & 12.13 & 8.64 & 6.64 & 5.63 \\
\hline Lilangeni per U.S. dollar (period average) & 6.94 & 8.60 & 10.52 & 7.56 & 6.45 \\
\hline
\end{tabular}

Sources: Central Bank of Swaziland; and staff projections.

1/ SACU: Southern African Customs Union. 
Table 30. Swaziland: Merchandise Exports, 2000-2003

(In millions of U.S. dollars)

\begin{tabular}{|c|c|c|c|c|}
\hline & 2000 & 2001 & 2002 & 2003 \\
\hline Sugar & 97 & 76 & 64 & 118 \\
\hline Wood pulp & 65 & 61 & 69 & 178 \\
\hline Asbestos & 3 & 1 & 0 & 0 \\
\hline Meat and meat products & 3 & 0 & 3 & 0 \\
\hline Canned fruit & 13 & 10 & 15 & 11 \\
\hline Citrus & 1 & 10 & 6 & 28 \\
\hline Coal & 7 & 2 & 6 & 25 \\
\hline Diamond & 0 & 0 & 0 & 0 \\
\hline Zippers & 13 & 12 & 10 & 20 \\
\hline Edible concentrates & 204 & 281 & 276 & 762 \\
\hline Wood and wood products & 22 & 19 & 17 & 0 \\
\hline Cottonseed and lint & 4 & 5 & 0 & 220 \\
\hline Chemicals & 0 & 0 & 0 & 0 \\
\hline Automobile & 0 & 0 & 0 & 0 \\
\hline Fruit squash & 14 & 9 & 6 & 20 \\
\hline Brake linings & 0 & 0 & 0 & 1 \\
\hline Cotton yarn & 16 & 10 & 14 & 0 \\
\hline Face bricks & 0 & 0 & 0 & 0 \\
\hline Footwear & 0 & 0 & 0 & 1 \\
\hline Other textiles & 18 & 50 & 149 & 0 \\
\hline Paper products & 18 & 8 & 8 & 26 \\
\hline Plastic products & 2 & 0 & 0 & 37 \\
\hline Refrigerators & 62 & 2 & 1 & 5 \\
\hline Ethanol & 3 & 0 & 9 & 0 \\
\hline Eucalyptus & 4 & 18 & 3 & 0 \\
\hline Television sets & 0 & 0 & 0 & 10 \\
\hline Windscreens & 0 & 0 & 0 & 1 \\
\hline Other food products & 35 & 23 & 37 & 0 \\
\hline Other/errors and omissions & 299 & 453 & 125 & -79 \\
\hline Total domestic exports & 903 & 1,051 & 818 & 1,384 \\
\hline Reexports & 7 & 6 & 217 & 100 \\
\hline Total exports, f.o.b. & 910 & 1,057 & 1,035 & 1,484 \\
\hline
\end{tabular}

Sources: Central Statistical Office; and Central Bank of Swaziland. 
Table 31. Swaziland: Sugar Exports by Volume, Value, and Unit Price, 2000-2004

\begin{tabular}{|c|c|c|c|c|c|}
\hline & 2000 & 2001 & 2002 & 2003 & 2004 \\
\hline & \multicolumn{5}{|c|}{ (In thousands of metric tons) } \\
\hline \multicolumn{6}{|l|}{ Volume } \\
\hline Non-SACU & 275 & 273 & 281 & 279 & 296 \\
\hline European Union (EU) & 183 & 169 & 153 & 147 & 158 \\
\hline United States & 16 & 16 & 16 & 16 & 16 \\
\hline Other & 76 & 88 & 112 & 116 & 122 \\
\hline SACU 1/ & 255 & 296 & 281 & 332 & 311 \\
\hline Total production $2 /$ & 528 & 501 & 583 & 628 & 598 \\
\hline & \multicolumn{5}{|c|}{ (In millions of emalangeni) } \\
\hline \multicolumn{6}{|l|}{ Value } \\
\hline Non-SACU & 673 & 662 & 956 & 787 & 745 \\
\hline EU & 542 & 498 & 731 & 596 & 576 \\
\hline United States & 42 & 50 & 68 & 44 & 34 \\
\hline Other & 89 & 114 & 157 & 147 & 135 \\
\hline SACU 1/ & 565 & 701 & 743 & 874 & 801 \\
\hline & \multicolumn{5}{|c|}{ (In U.S. dollars per metric ton) } \\
\hline \multicolumn{6}{|l|}{ Average unit price } \\
\hline Non-SACU & 353 & 282 & 324 & 373 & 390 \\
\hline EU & 427 & 343 & 454 & 536 & 567 \\
\hline United States & 378 & 363 & 404 & 364 & 329 \\
\hline Other & 170 & 151 & 133 & 168 & 172 \\
\hline SACU 1/ & 319 & 275 & 251 & 348 & 399 \\
\hline & \multicolumn{5}{|c|}{ (In emalangeni per metric ton) } \\
\hline \multicolumn{6}{|l|}{ Average unit price } \\
\hline Non-SACU & 2,450 & 2,425 & 3,402 & 2,821 & 2,517 \\
\hline EU & 2,959 & 2,947 & 4,778 & 4,054 & 3,657 \\
\hline United States & 2,625 & 3,125 & 4,250 & 2,750 & 2,125 \\
\hline Other & 1,179 & 1,295 & 1,402 & 1,267 & 1,107 \\
\hline SACU 1/ & 2,216 & 2,368 & 2,644 & 2,633 & 2,576 \\
\hline
\end{tabular}

Source: Swaziland Sugar Association.

1/ The Southern African Customs Union (SACU) accounts for all sales to SACU countries and includes sales of sugar within Swaziland (normally about 10-15 percent of SACU sales).

$2 /$ The difference between total production and total exports represents a change in stock. 
Table 32. Swaziland: Other Principal Exports by Volume, Value, and Unit Price, 2000-2004

\begin{tabular}{|c|c|c|c|c|c|}
\hline & 2000 & 2001 & 2002 & 2003 & 2004 \\
\hline & \multicolumn{5}{|c|}{ (In thousands of metric tons) } \\
\hline \multicolumn{6}{|l|}{ Volume } \\
\hline Wood pulp & 133 & 186 & 198 & 168 & 181 \\
\hline Asbestos & 13 & 3 & $\ldots$ & $\ldots$ & $\ldots$ \\
\hline Citrus fruits & 101 & 100 & 90 & 75 & 71 \\
\hline Canned fruits & 20 & 27 & 26 & 26 & 25 \\
\hline & \multicolumn{5}{|c|}{ (In millions of emalangeni) } \\
\hline \multicolumn{6}{|l|}{ Value } \\
\hline Wood pulp & 446 & 516 & 638 & 425 & 446 \\
\hline Asbestos & 21 & 5 & $\ldots$ & $\ldots$ & $\ldots$ \\
\hline Citrus fruits & 71 & 83 & 60 & 81 & 81 \\
\hline Canned fruits & 87 & 112 & 149 & 117 & 110 \\
\hline & \multicolumn{5}{|c|}{ (In U.S. dollars per metric ton) } \\
\hline \multicolumn{6}{|c|}{ Average unit price } \\
\hline Wood pulp & 485 & 324 & 308 & 335 & 382 \\
\hline Asbestos & 234 & 194 & $\ldots$ & $\ldots$ & $\ldots$ \\
\hline Citrus fruits & 102 & 97 & 64 & 102 & 136 \\
\hline Canned fruits & 629 & 522 & 528 & 604 & 682 \\
\hline & \multicolumn{5}{|c|}{ (In emalangeni per metric ton) } \\
\hline \multicolumn{6}{|c|}{ Average unit price } \\
\hline Wood pulp & 3,353 & 2,774 & 3,222 & 2,534 & 2,464 \\
\hline Asbestos & 1,615 & 1,667 & $\ldots$ & $\ldots$ & $\ldots$ \\
\hline Citrus fruits & 703 & 830 & 667 & 768 & 877 \\
\hline Canned fruits & 4,350 & 4,480 & 5,519 & 4,564 & 4,400 \\
\hline
\end{tabular}

Source: Central Bank of Swaziland. 
Table 33. Swaziland: Merchandise Imports, 2001-2003

(In millions of U.S. dollars, unless otherwise indicated)

\begin{tabular}{lrrr}
\hline & 2001 & 2002 & 2003 \\
& & & \\
\hline & 132 & 132 & 203 \\
Food and live animals & 20 & 18 & 24 \\
Beverages and tobacco & 33 & 27 & 40 \\
Inedible crude materials & 97 & 113 & 143 \\
Minerals, fuels, and lubricants & 10 & 9 & 14 \\
Animal and vegetable oils and fat & 112 & 104 & 190 \\
Chemicals and chemical products & 148 & 146 & 222 \\
Manufactures classified by material & 197 & 202 & 452 \\
Machinery and transport equipment & 97 & 91 & 141 \\
Miscellaneous manufactures & $\ldots$ & $\ldots$ & 14 \\
Commodities not classified by kind & & & \\
& $\ldots$ & $\ldots$ & 1,442 \\
Total imports, c.i.f. & $\ldots$ & $\ldots$ & \\
Total imports, f.o.b. & & & \\
Imports f.o.b./imports c.i.f. & & & \\
\end{tabular}

Source: Central Bank of Swaziland. 
Table 34: Swaziland: Services and Income Account, 2001-2004

(In millions of U.S. dollars)

\begin{tabular}{|c|c|c|c|c|}
\hline & 2001 & 2002 & 2003 & 2004 \\
\hline Nonfactor services: credit & 114.0 & 72.4 & 156.5 & 482.3 \\
\hline Swaziland Railways & 8.2 & 5.6 & 12.5 & 12.8 \\
\hline Other shipment and transportation & 4.9 & 7.3 & 21.5 & 26.0 \\
\hline Travel & 30.6 & 42.6 & 101.0 & 123.4 \\
\hline Other official & 2.2 & 3.4 & 7.0 & 12.8 \\
\hline Other Private & 68.2 & 13.5 & 14.4 & 307.4 \\
\hline Nonfactor services: debit & -183.3 & -142.8 & -205.7 & -534.7 \\
\hline Shipment and tansp. & -42.1 & -33.8 & -77.0 & -88.9 \\
\hline Travel & -46.7 & -39.4 & -5.8 & 5.6 \\
\hline Other official & -16.1 & -7.9 & -12.0 & -12.6 \\
\hline Other Private & -78.4 & -61.7 & -110.9 & -438.8 \\
\hline Factor income: credit & 157.4 & 145.3 & 143.5 & 160.8 \\
\hline Investment income & 83.5 & 83.5 & 59.5 & 53.6 \\
\hline Official & 82.2 & 80.0 & 57.3 & 50.1 \\
\hline Interest & 82.2 & 80.0 & 57.3 & 50.1 \\
\hline Private (incl. Property income) $1 /$ & 1.3 & 3.6 & 2.2 & 3.1 \\
\hline Labour income & 73.9 & 61.7 & 84.0 & 107.2 \\
\hline Factor income: debit & -97.6 & -195.1 & -147.2 & -128.9 \\
\hline Investment income & -54.6 & -140.5 & -83.6 & -63.6 \\
\hline Official \& Govnt. guaranteed & -13.9 & -15.0 & -25.6 & -15.6 \\
\hline Private & -40.7 & -125.6 & -58.1 & -48.1 \\
\hline Property income 1/ & -43.0 & -54.5 & -60.0 & -61.7 \\
\hline Labour income & 0.0 & 0.0 & -3.6 & -3.6 \\
\hline Services and income: credit & 271.4 & 217.6 & 300.0 & 643.1 \\
\hline Services and income: debit & -280.9 & -337.9 & -352.9 & -663.6 \\
\hline Services (net) $1 /$ & -69.3 & -70.4 & -49.2 & -52.4 \\
\hline Factor income (net) 1/ & 59.9 & -49.8 & -3.7 & 31.9 \\
\hline Total (net) & -9.5 & -120.2 & -52.9 & -20.4 \\
\hline
\end{tabular}

Source: Central Bank of Swaziland

1/ The Table shows the Central Bank's classification. In Tables 32 and 33, property income - mostly royalties and license fees is grouped under services, so that the totals for services and factor income differ by a corresponding amount. 
Table 35. Swaziland: Financial Account, 2001-2004

(In millions of U.S. dollars)

\begin{tabular}{|c|c|c|c|c|}
\hline & 2001 & 2002 & 2003 & 2004 \\
\hline Direct investment (net) & 46.3 & 84.9 & -70.7 & 65.0 \\
\hline Direct investment in Swaziland & 28.5 & 78.7 & -61.0 & 68.6 \\
\hline Equity capital & 38.2 & -10.1 & -3.8 & -4.8 \\
\hline Reinvestment of earnings & -0.2 & 65.3 & -25.7 & 36.7 \\
\hline Other long-term capital & 18.3 & -28.9 & 15.9 & 19.4 \\
\hline Other short-term capital & -27.7 & 52.4 & -47.4 & 17.3 \\
\hline Direct investment abroad & 17.7 & 6.1 & -9.8 & -3.6 \\
\hline Equity capital & 0.0 & 0.4 & 0.4 & 0.0 \\
\hline Reinvestment of earnings & -0.6 & 0.0 & 0.0 & 0.0 \\
\hline Other long-term capital & -0.1 & 0.1 & 0.3 & 0.0 \\
\hline Other short-term capital & 18.4 & 5.7 & -10.4 & -3.6 \\
\hline Long-term capital (net) & -231.1 & -94.6 & -45.0 & -45.2 \\
\hline Public sector (net) & 4.3 & 6.3 & -2.2 & 6.9 \\
\hline Loan drawings (liability) & 16.0 & 20.5 & 15.2 & 28.3 \\
\hline Loan repayments (liability) & -11.7 & -14.2 & -17.4 & -21.4 \\
\hline Sasol Funds (assets) & 0.0 & 0.0 & 0.0 & 0.0 \\
\hline Private sector (net) & -235.4 & -101.0 & -42.8 & -52.1 \\
\hline Private sector (liability) & -27.7 & -31.2 & -1.1 & 0.3 \\
\hline Corporate equity securities & -2.5 & -2.0 & -0.1 & -0.3 \\
\hline Loans (drawings and repayments) & -25.1 & -29.2 & -1.0 & 0.6 \\
\hline Private sector (assets) & -207.7 & -69.7 & -41.8 & -52.4 \\
\hline Corporate equity securities & -0.1 & 0.0 & 0.0 & 0.2 \\
\hline Debt securities (money markets) & -5.3 & 3.9 & -0.3 & -10.9 \\
\hline Loans (drawings and repayments) & 0.0 & 0.0 & -5.3 & 3.6 \\
\hline Other assets including pension funds & -202.3 & -73.7 & -36.1 & -45.3 \\
\hline Short-term capital (net) & 43.9 & -83.9 & 89.1 & -130.2 \\
\hline Public sector (net) & 15.6 & -33.9 & -51.6 & -103.7 \\
\hline Received under Southern African Customs Union (SACU) (liability) & 205.2 & 146.6 & 222.7 & 308.0 \\
\hline Monetary authority currency/deposits (liability) & 14.8 & -9.8 & -2.7 & -50.3 \\
\hline Other liabilities & 0.0 & 0.0 & 0.0 & 0.0 \\
\hline Receivable under SACU & -204.4 & -170.7 & -271.5 & -361.4 \\
\hline Private sector (net) & 28.3 & -50.0 & 140.7 & -26.5 \\
\hline Banks (net) & -37.3 & 14.4 & 32.5 & -3.2 \\
\hline Currency and deposits (assets) & -27.8 & 9.6 & 35.7 & -1.6 \\
\hline Other liabilities (liability) & -9.5 & 4.8 & -3.3 & -1.7 \\
\hline Other (net) & 65.6 & -64.4 & 108.2 & -23.3 \\
\hline Trade credit (liability) & 2.0 & -6.8 & 2.0 & -1.3 \\
\hline Other liabilities (liability) & -2.1 & -6.5 & -12.8 & -0.9 \\
\hline Trade credit (assets) & -7.5 & -13.0 & 100.7 & -2.2 \\
\hline Currency holdings & 73.1 & -38.1 & 18.3 & -18.9 \\
\hline Other (unrecorded) & 73.2 & 29.1 & $\ldots$ & $\ldots$ \\
\hline Financial account balance & -67.7 & -64.5 & -28.5 & -23.3 \\
\hline
\end{tabular}


Table 36. Swaziland: Public Sector External Debt, 2000/01-2004/05 1/

(In millions of U.S. dollars)

\begin{tabular}{|c|c|c|c|c|c|}
\hline & $2000 / 01$ & $2001 / 02$ & $2002 / 03$ & $2003 / 04$ & $2004 / 05$ \\
\hline Multilateral loans & 173 & 155 & 160 & 195 & 283 \\
\hline IBRD & 29 & 10 & 9 & 16 & 22 \\
\hline IDA & 5 & 4 & 4 & 4 & 4 \\
\hline Arab League & 0 & 0 & 0 & 0 & 0 \\
\hline United Nations & 0 & 0 & 0 & 0 & 0 \\
\hline African Development Bank/Fund & 125 & 128 & 123 & 142 & 223 \\
\hline European Development Fund/European Investment Bank & 9 & 9 & 21 & 30 & 31 \\
\hline IMF Trust Fund & 0 & 0 & 0 & 0 & 0 \\
\hline International Fund for Agricultural Development & 6 & 4 & 3 & 3 & 3 \\
\hline Economic Development for Equatorial and Southern Africa & 0 & 0 & 0 & 0 & 0 \\
\hline Bilateral loans & 47 & 49 & 104 & 171 & 165 \\
\hline United Kingdom & 2 & 1 & 1 & 1 & 1 \\
\hline Germany & 23 & 21 & 11 & 29 & 29 \\
\hline United States & 8 & 7 & 7 & 7 & 6 \\
\hline Denmark & 6 & 5 & 6 & 6 & 5 \\
\hline Canada & 0 & 0 & 0 & 0 & 0 \\
\hline Other & 8 & 14 & 79 & 128 & 124 \\
\hline Financial institutions & 39 & 33 & 24 & 37 & 38 \\
\hline Other & 0 & 0 & 0 & 0 & 0 \\
\hline Total & 258 & 237 & 289 & 403 & 486 \\
\hline
\end{tabular}

Source: Ministry of Finance.

1/ Fiscal year runs from April 1 to March 31. 
Table 37. Swaziland: Public Sector External Debt Service, 2000/01-2004/05 1/ (In millions of emalangeni)

\begin{tabular}{|c|c|c|c|c|c|}
\hline & $2000 / 01$ & $2001 / 02$ & $2002 / 03$ & $2003 / 04$ & $2004 / 05$ \\
\hline Multilateral loans & 99.0 & 142.1 & 155.0 & 141.0 & 115.0 \\
\hline Interest & 38.6 & 59.2 & 67.0 & 63.0 & 49.0 \\
\hline Principal & 60.5 & 82.9 & 88.0 & 78.0 & 66.0 \\
\hline Bilateral loans & 39.7 & 65.3 & 68.0 & 70.0 & 35.0 \\
\hline Interest & 16.1 & 13.4 & 20.0 & 36.0 & 7.0 \\
\hline Principal & 23.6 & 51.9 & 48.0 & 34.0 & 28.0 \\
\hline Financial institutions & 2.5 & 26.1 & 26.0 & 10.0 & 64.0 \\
\hline Interest & 2.5 & 22.7 & 21.0 & 10.0 & 64.0 \\
\hline Principal & 0.0 & 3.3 & 5.0 & 0.0 & 0.0 \\
\hline Total & 141.2 & 233.5 & 249.0 & 221.0 & 214.0 \\
\hline Interest & 57.1 & 95.4 & 108.0 & 109.0 & 120.0 \\
\hline Principal & 84.1 & 138.1 & 141.0 & 112.0 & 94.0 \\
\hline
\end{tabular}

Source: Ministry of Finance.

1/ Fiscal year runs from April 1 to March 31. 
Table 38. Swaziland: Exports and Share of South Africa, 2003

\begin{tabular}{cc}
2003 \\
\hline Exports to \\
\hline (In thousands of U.S. dollars)
\end{tabular}

Share of

Exports to

South Africa

(In percent)

Standard International Trade Classification (SITC)

Food and beverages

Citrus fruit

Canned fruit and fruit juices

Sugar, mainly raw

Nonalcoholic beverages

Crude materials

Coke and wood

Pulp and waste paper

Manufactures classified by material

Paper products

Textiles and yarns

Nonmetallic minerals

Machinery and transport equipment

General industrial machinery

Other machinery and equipment

Miscellaneous manufactured articles

Furniture and parts thereof

Apparel and clothing

Footwear
181,965

28,369

35,818

117,718

61

220,113

42,049

178,064

65,519

36,679

28,827

13

123,579

3,783

8,575

703,910

27,006

676,578

326

188,986

$1,484,073$
Total, f.o.b.

Other
132,059

13,782

15,953

102,325

50

98,323

35,317

63,006

54,867

35,616

19,251

8

7,979

2,158

5,820

488,046

13,657

474,389

159

151,179

Source: Department of Customs and Excise, Swaziland.
80

73

49

45

87

82

45

84

35

84

97

67

60

6

57

68

69

51

70

49

63 
Table 39. Swaziland: Main Imports and Share of South Africa, 2003

\begin{tabular}{|c|c|c|c|}
\hline & $\frac{\text { Total Imports }}{\text { (In thousand }}$ & $\begin{array}{l}\text { Imports from } \\
\text { South Africa } \\
\text { U.S. dollars) }\end{array}$ & $\begin{array}{l}\text { Share of } \\
\text { Imports from } \\
\text { South Africa } \\
\text { (In percent) }\end{array}$ \\
\hline \multicolumn{4}{|c|}{ Standard International Trade Classification (SITC) } \\
\hline Food and beverages & 174,915 & 167,344 & 85 \\
\hline Live animals & 11,476 & 11,474 & 100 \\
\hline Dairy products & 19,830 & 18,221 & 92 \\
\hline Vegetables & 21,319 & 20,457 & 96 \\
\hline Cereals & 5,473 & 4,256 & 78 \\
\hline Flour, malt, and gluten & 59,028 & 56,654 & 96 \\
\hline Sugar & 0 & 0 & 0 \\
\hline Bread and pasta & 18,735 & 18,733 & 100 \\
\hline Juice and jams & 9,947 & 9,874 & 99 \\
\hline Ice cream, sauces, and soups & 8,050 & 8,003 & 99 \\
\hline Beverages & 21,057 & 19,672 & 93 \\
\hline Chemicals and fuels & 306,101 & 298,000 & 95 \\
\hline Inorganic chemicals & 16,428 & 16,160 & 98 \\
\hline Pharmaceuticals & 24,039 & 20,753 & 86 \\
\hline Fertilizers & 13,706 & 13,700 & 100 \\
\hline Toiletries and perfumes & 54,350 & 54,219 & 100 \\
\hline Soaps, polishes, and wax & 17,148 & 17,120 & 100 \\
\hline Gelatin and adhesives & 1,560 & 1,329 & 85 \\
\hline Plastics & 42,658 & 38,611 & 91 \\
\hline Fuels (coal and petroleum) & 136,212 & 136,108 & 100 \\
\hline Manufactures classified by materials & 135,173 & 129,452 & 97 \\
\hline Rubber and products thereof & 16,109 & 15,997 & 99 \\
\hline Wood and products thereof & 36,861 & 36,689 & 100 \\
\hline Paper products & 71,874 & 67,220 & 94 \\
\hline Newspaper, printed & 968 & 966 & 100 \\
\hline Special woven fabrics & 2,382 & 2,355 & 99 \\
\hline Iron and steel products & 5,924 & 5,174 & 87 \\
\hline Aluminum products & 1,054 & 1,052 & 100 \\
\hline \multicolumn{4}{|l|}{ Nonmetallic minerals } \\
\hline Machinery, transport equipment, and others & 541,750 & 483,820 & 89 \\
\hline Nonelectric machinery & 250,375 & 238,220 & 95 \\
\hline Electric machinery & 56,257 & 52,274 & 93 \\
\hline Vehicles & 109,938 & 105,960 & 96 \\
\hline Clothing & 90,980 & 53,338 & 59 \\
\hline Footwear & 13,491 & 13,396 & 99 \\
\hline Furniture & 20,708 & 20,631 & 100 \\
\hline Subtotal & $1,157,939$ & $1,078,616$ & 93 \\
\hline Other & 284,202 & 204,314 & 72 \\
\hline Total & $1,442,142$ & $1,282,930$ & 89 \\
\hline
\end{tabular}

Source: Department of Customs and Excise, Swaziland. 
Table 40. Swaziland: Summary of the Tax System as of December 31, 2005

(All amounts in emalangeni)

\begin{tabular}{llll}
\hline Tax & Nature of Tax & Exemptions and Deductions
\end{tabular}

A. Central government

1. Taxes on net income and profits

\subsection{Taxes on companies}

1.11 Company income tax (normal tax). Income Tax Order, 1975, as amended.
Annual income tax is levied on taxable income derived from sources in Swaziland, or deemed to be in Swaziland, by all companies, foreign or domestic, public or private. Agricultural cooperatives, insurance societies, and public utility companies are also considered companies for taxation purposes. Taxable income is defined as gross income (excluding capital receipts, and foreign and exempt income) less allowable deductions (including loss offsets) incurred in the process of production in Swaziland. The assessment year ends June 30 and tax (less provisional tax payments detailed below) is payable annually. Company tax legislation is integrated with personal income tax legislation. Consequently, where exemptions, deductions, and allowances normally appropriate for persons are appropriate for a particular company, these may be claimed (see 1.21 below). For farming companies, net change in livestock and produce held is deemed income (except for companies that opted otherwise under previous tax laws) and will be valued at purchase price or current market prices, whichever is lower.
Exemptions include, inter alia, dividend receipts of companies, receipts and accruals (including those from investments) of life insurance companies, pension benefits or provident funds. Also excluded are the noninvestment profits of societies and associations that are derived solely through transactions with individual members. (a) Companies: 30 percent of taxable income.

(b) Companies granted development approval order: 10 percent of taxable income. 
Table 40. Swaziland: Summary of the Tax System as of December 31, 2005 (continued) (All amounts in emalangeni)

\begin{tabular}{l}
\hline Tax \\
\hline 1.11 Company income tax \\
(normal tax). Income Tax \\
Order, 1975, as amended. \\
(continued)
\end{tabular}

Nature of Tax

Exemptions and Deductions

Rates

Provisional tax: a system of

provisional taxation is in operation in

respect of all companies, directors of

private companies, and any person

whose income, other than

remuneration under the PAYE (pay-

as-you-earn) scheme, exceeds

E 1,000 per annum. Provisional taxpayers pay provisional tax on account of their final liability for normal tax for the year. The advance payments are to be made as follows:

(a) on or before the last day of the six months of the year of assessment, one-half of the tax payable on the estimated income for the year; and (b) on or before the last day of the year of assessment, the total taxes payable on the estimated income for the year less the amount of the previous provisional tax payment. The estimated income for the year will normally not be less than the taxable income in the preceding year of assessment. In the case of a company, the year of assessment will be the year in respect of which its financial accounts are drawn up. Farmers are required to make one payment of provisional tax on or before the end of the year of assessment on the estimated income for the year. As an introductory measure, a "phasing-in" period of five years is provided for payment of provisional tax. One-fifth of the provisional tax due for 1985/86 would be payable in that year and so on until full provisional tax payments are due in 1990/91. In terms of the provisions of the Income Tax (Amendment) Act of 1994, all companies that are provisional taxpayers are required, in addition to the two existing provisional tax payments mentioned above, to make a third payment for year of assessment. This final payment is due six months after the close of the year of assessment, by which time the company will be in a position to accurately estimate its taxable income.
A new business engaged in a

manufacturing industry that is not already in existence in Swaziland or, with effect from July 1, 1987, any business that is predominantly engaged in exporting goods from Swaziland is exempt from normal tax for the first five years unless the cumulative taxable income less cumulative local wage bill exceeds 150 percent of the value of the assets, in which case the excess shall be taxed. The Minister of Finance makes the final decision on whether a business is new, engaged in a manufacturing industry, is not already in existence in Swaziland, or is predominantly engaged in exporting goods from Swaziland.

Where the Minister of Finance is satisfied that a new business is beneficial to the development of the economy, he may, with prior approval of cabinet, declare that business a development enterprise, and he may issue a "development approval order" in respect of that business's granting additional tax concessions to it.

Deductions allowed include expenditures and losses incurred in the production of income (excluding capital expenditures and dividend payments), interest charges, "reasonable" depreciation allowances for plants, and 4 percent for buildings used in production, along with actual expenditures on repairs and maintenance. Any grant made to the University of Swaziland for the purpose of the university's undertaking capital projects in the form of buildings, fittings, and furniture, as well as other items associated with capital assets needed for the development of the university is exempt. An amount spent by a company as direct "listing" fees on the Swaziland Stock Exchange, subject to the proviso that only one-third of the expense is claimable in the year of expense; the balance is spread equally in the next two years. 
Table 40. Swaziland: Summary of the Tax System as of December 31, 2005 (continued)

(All amounts in emalangeni)

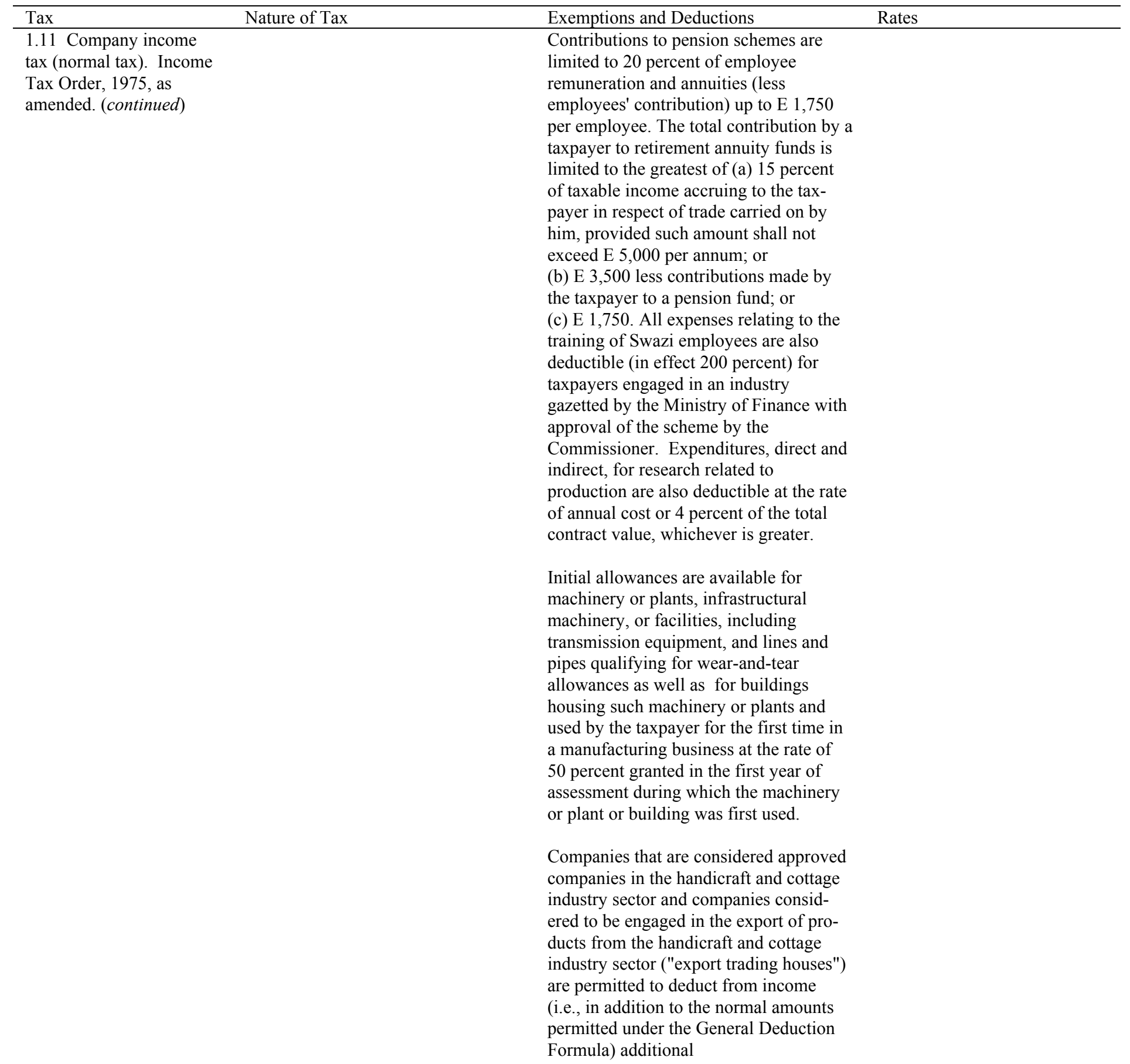


Table 40. Swaziland: Summary of the Tax System as of December 31, 2005 (continued)

(All amounts in emalangeni)

\begin{tabular}{|c|c|}
\hline Nature of Tax & Exemptions and Deductions \\
\hline \multirow[t]{3}{*}{$\begin{array}{l}\text { 1.11 Company income tax } \\
\text { (normal tax). Income Tax } \\
\text { Order, 1975, as amended. } \\
\text { (concluded) }\end{array}$} & $\begin{array}{l}\text { amounts of } 133 \text { percent (for the cottage } \\
\text { industry) and } 150 \text { percent ("approved } \\
\text { export trading houses") in respect of } \\
\text { "approved export promotion } \\
\text { expenditure," as defined. The additional } \\
\text { expenditure allowance is subject to the } \\
\text { company's achieving an increase in } \\
\text { volume of exports in the subsequent } \\
\text { year. }\end{array}$ \\
\hline & $\begin{array}{l}\text { Contributions, whether in cash or in } \\
\text { kind, made during the year of assessment } \\
\text { toward any national disaster scheme } \\
\text { established by the government. }\end{array}$ \\
\hline & $\begin{array}{l}\text { Farming: special ( } 100 \text { percent) } \\
\text { deductions (not exceeding } 30 \text { percent of } \\
\text { gross income) are allowable for a variety } \\
\text { of on-farm expenditures (e.g., irrigation } \\
\text { and fencing). Where these deductions are } \\
\text { made, initial and depreciation allowances } \\
\text { are not allowable. }\end{array}$ \\
\hline
\end{tabular}

1.12 Casino tax. Casino Tax Act, 1963, (Act No. 56, 1963, as amended).
With effect from July 1, 1985, it is composed of an annual license fee and of a levy based on a percentage of the gross gaming room takings of the licensee less any amount paid out as winnings to casino patrons and is payable on an annual basis. A licensee is liable to normal tax.
Rates

(a) The annual license fee is E 2,000.

(b) A levy payable on the gross gaming room takings of the licensee less any amount paid out as winnings to casino patrons, as follows:

- In respect of the first year of operation of the casino, no levy shall be paid.

- In respect of the second, third, fourth, fifth, and sixth years of operation of the casino, a levy of 2.0 percent shall be paid.

- In respect of the seventh year and subsequent years of operation, a levy of 4.5 percent shall be paid. 
Table 40. Swaziland: Summary of the Tax System as of December 31, 2005 (continued) (All amounts in emalangeni)

\begin{tabular}{l} 
Tax \\
\hline 1.21 Individual income \\
tax (normal tax). Income \\
Tax Order, 1975, as \\
amended.
\end{tabular}

Nature of Tax

This is payable, subject to the

deductions and exemptions noted, on income received by or accruing to all persons from sources within Swaziland or deemed to be within Swaziland. Tax is payable on assessed "taxable income," which is equal to gross income (excluding capital receipts and exempt income) less losses and allowable deductions. Taxable income includes, inter alia, annuities, wages and salaries, rent investment income, and benefits in kind (e.g., free housing). After the calculation of taxable income and, hence, tax payable, certain "tax abatements" may be deducted where applicable. With effect from July 1, 1991, both men and women are subject to a uniform system of taxation. (See below under "rates.")

Employees are subject to a monthly withholding at source; other taxpayers are assessed annually. Nonresidents are liable for income tax on income earned in Swaziland (including benefits in kind received for services rendered); however, dividends and interest payments are subject to special taxes (see 1.31 and 1.32). Personal income tax legislation is integrated with company tax legislation. Consequently, where exemptions, deductions, and allowances normally appropriate for companies are applicable to persons (e.g., owneroccupied farms), these may be claimed. For farmers, net change in produce and livestock held is deemed income (except for those farmers who opted otherwise under previous tax laws) and will be valued at the lesser of the purchase price or current market price (if purchased), and at standard book valuation otherwise.

A system of provisional tax is in operation in respect of self-employed persons and other individuals whose income, other than remuneration subject to PAYE, exceeds

Exemptions and Deductions Rates

Exemptions include, inter alia, every person who is ordinarily resident in Swaziland whose taxable income in one year of assessment does not exceed E 20,000 per annum. Other exemptions include the following: salaries of U.K. and South African civil servants; consular personnel not permanent residents of Swaziland; war pensions and gratuities; the first E 1,000 of interest income received by or accrued to an individual from a deposit in a financial institution; interest received by nonresidents from Swaziland government securities and bonds; and capital sums due from a provident fund or benefit fund (pension one third of the total value of the annuity to which any employee becomes entitled may be commuted for a single payment); capital sums in commutation of a retirement annuity; gratuities to a maximum of 25 percent of total remuneration in respect of services rendered prior to 1 July 2001 . Severance allowance or notice pay payable under the Employment Act to an employee on the termination of his services is exempt from normal tax. The first E 30,000 received by or accrued to an individual on retrenchment or retirement.

$\begin{array}{lr}\text { Rates } & \\ \begin{array}{c}\text { Taxable income } \\ \text { (in emalangeni) }\end{array} & \begin{array}{r}\text { Marg } \\ \text { tax }\end{array} \\ & \\ \text { (In percent) } & \\ 0-20,000 & 0 \\ 20,000-30,000 & 12 \\ 30,000-45,000 & 20 \\ 45,001-60,000 & 25 \\ 60,001-75,000 & 30 \\ 75,000 & 33 \\ \text { Tax credit E2400 p.a. }\end{array}$

Trust income

33 percent of taxable income

Rates of normal tax in the case of a retiring or redundant individual

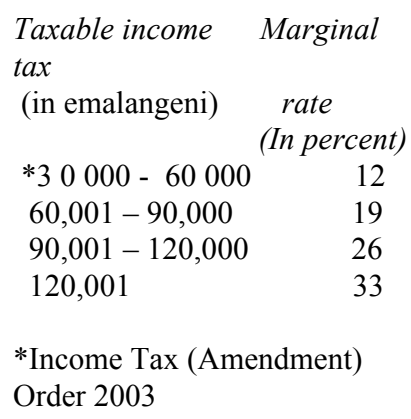


Table 40. Swaziland: Summary of the Tax System as of December 31, 2005 (continued)

(All amounts in emalangeni)

\begin{tabular}{ll}
\hline Tax & Nature of Tax \\
\hline $\begin{array}{l}\text { 1.21 Individual income } \\
\text { tax (normal tax). Income }\end{array}$ & E 1,000 per annum (see 1.11 above). \\
Tax Order, 1975, as & One of the important amendments \\
amended. (concluded) & (Ameduced in the Income Tax \\
& the introduction of the Final \\
& Deduction System (FDS) for \\
& employees, which came into \\
& operation on July 1, 1993. FDS \\
& constitutes a final liability to tax and \\
& is related to a full year of assessment. \\
& All employees, no matter how much \\
& they earn, are subject to the FDS, \\
& provided they have not derived any \\
other taxable income during the year & of assessment. Such employees are \\
& not required to furnish an income tax \\
& return if the income consists solely of \\
employment income that is subject to & FDS.
\end{tabular}

Exemptions and Deductions

Rates

Dividends received by nonresidents (see

1.32 below).

Deductions include, in addition to those

for companies (1.11 above) where

appropriate, employee contributions to

pension funds (maximum E 1,750 where

the pension fund is not established by

law).

Death, accident, sickness, or unemployment insurance and contributions to provident and benefit funds (other than a medical aid fund) are also deductible at a rate of 10 percent to a maximum of $\mathrm{E}$ 180. To receive this deduction on death, accident, and sickness insurance premiums on policies entered after July 1, 1974, the policy must be with the Swaziland Royal Insurance Corporation.

1.22 Graded tax. Graded Tax Act of 1968, as amended.

1.31 Tax on nonresidents' interest receipts. Income Tax Order, 1975, as amended.
This tax is payable by all persons (apart from the exemptions noted) resident or domiciled in Swaziland, and is thus akin to a head tax. Tax is determined on the basis of gross income and is payable annually for all except for employees whose deductions are made monthly at source.

This tax is payable by persons or the estate of a deceased person not resident in Swaziland or a company not registered in Swaziland on accrued interest where the debtor (person or company) is domiciled in Swaziland. The recipient is legally liable for the payment of this tax within 14 days of the accrual of interest, but it is normally paid by the debtor and deducted from interest remitted.
Exemptions include all persons under the apparent age of 18, women earning less than E 15 per month, visitors, students, and the chronically ill.

Exemptions: interest on loans specifically exempt by government (usually government and other public body loans); building society shares; interest from loans to agricultural cooperatives and public utilities established by parliament; interest received by church, charitable, or educational organizations; and interest amounting to E 20 or less in a full tax year. Also, interest on importers' bills or notes is exempt where these have been handled through the banking system.
Tax due is determined on the basis of gross income as shown below:

Gross income Tax payable
E 0 - E 299
E 4.20
E 300 - E 449
E 6.00
E 450 - E 600
E 12.00
Over E 600
E 18.00

Tax is payable at 10 percent of the interest accrued. 
Table 40. Swaziland: Summary of the Tax System as of December 31, 2005 (continued) (All amounts in emalangeni)

\begin{tabular}{|c|c|}
\hline Tax & Nature of Tax \\
\hline $\begin{array}{l}\text { 1.32 Tax on nonresident } \\
\text { shareholders. Income Tax } \\
\text { Order, 1975, as amended. }\end{array}$ & $\begin{array}{l}\text { This tax is payable by persons or the } \\
\text { estate of a deceased person not } \\
\text { resident in Swaziland or any } \\
\text { company not registered in Swaziland } \\
\text { on dividends received from a } \\
\text { company domiciled in Swaziland. } \\
\text { This tax is payable on both interim } \\
\text { and final dividends and is due within } \\
30 \text { days. Legal liability for payment } \\
\text { resides with the recipient, but is } \\
\text { normally paid by the payer and } \\
\text { deducted from remitted dividends. }\end{array}$ \\
\hline
\end{tabular}

1.33 Tax on branch profits.

1.34 Tax on nonresident contractors. Income Tax Order, 1975, as amended.

1.35 Tax on nonresident. for Swaziland source services contract. Income Tax Order, 1975, as amended.

Exemptions and Deductions

Exemptions include dividends paid by agricultural cooperatives and dividends received by church, charitable, or educational institutions, as well as dividends accruing to a nonresident shareholder, which the government has, in terms of a written undertaking, exempted from tax.

Tax is payable on the deemed repatriated income of a branch of a nonresident company.

No exemptions
No exemptions.

This tax is payable by every person who makes payment to a nonresident person under an agreement relating to construction operations. The tax is deducted from each payment made to the nonresident. The nonresident is not relieved from any obligations to furnish returns for income tax and any assessment raised on the nonresident for income tax will be credited with the nonresident contractors' tax that has been paid on his behalf.

Tax is payable by all nonresident persons on amounts derived by the nonresident from performance of services that gives rise to Swaziland source income. The tax is payable on gross amount at source by withholding. The tax is a final tax.
No exemptions.
Rates

Tax is payable at the rate of $12^{1} / 2$ percent where dividends are payable to a company incorporated (but not a branch of company headquartered in a third country) in Botswana, Lesotho, Namibia, and South Africa.

For all other destinations, tax is payable at the rate of 15 percent.

Tax is payable at the rate of 15 percent.

A rate of $12^{1 / 2}$ percent where repatriated profits are payable to a company incorporated (but not a branch of company headquartered in a third country) in Botswana, Lesotho, Mozambique, Namibia, and South Africa

Tax is payable at the rate of 15 percent.
Tax is payable at the rate of 15 percent. 
Table 40. Swaziland: Summary of the Tax System as of December 31, 2005 (continued)

(All amounts in emalangeni)

\begin{tabular}{lll}
\hline Tax & Nature of Tax & Exemptions and Deductions \\
\hline $\begin{array}{l}\text { 1.36 Tax on nonresident } \\
\text { entertainers and sport- }\end{array}$ & This tax is payable by all nonresident & There are no exemptions \\
smen. Income Tax Order, & perform in Swaziland. The tax is \\
1975, as amended. & payable on remuneration or gross \\
& receipts of any theatrical, musical, or \\
& sports performance where these \\
& receipts are received directly by the \\
& performers concerned. Legal \\
& liability for payment resides with the \\
& recipients. However, the payer is \\
& legally required to withhold the tax \\
& due from any remuneration payable.
\end{tabular}

1.37 Tax on royalties and management charge paid to nonresident persons. Income Tax Order, 1975, as amended.

1.38 Tax on interest paid to residents. Income Tax Order, 1975, as amended.

1.39 Withholding tax on dividends paid to resident persons

1.40 Tax clearance certificates.

2. Social security contributions
This tax is payable by nonresident persons on gross amount of any royalty and management charge derived from a source in Swaziland.

The tax is withheld at source and it is a final tax.

This tax is payable by residents on the gross amount of interest derived from every financial institution. The tax is withheld at source. It is a final tax.

Tax is payable by a person (other than a company) resident or carrying on business in Swaziland.

A system of tax clearance is in operation. In terms of a gazette regulation published recently, tax clearance is presently needed for the issue, renewal, or transfer of any license, other than renewal of motor vehicle licenses, or similar document relating to any trade, business, profession, or vocation; the transfer of immovable property or any endorsement to any title deed having the effect of transferring property; the registration or deregistration of a company; first registration of motor vehicles in Swaziland; and the tendering for the provision of goods or services to the government or a parastatal body, in excess of E 5,000. None.
There are no exemptions.

There are no exemptions.

There are no exemptions.
Tax is payable at the rate of 15 percent.

Tax is payable at the rate of 10 percent.

Tax is payable at the rate of 10 percent. 
Table 40. Swaziland: Summary of the Tax System as of December 31, 2005 (continued) (All amounts in emalangeni)

\begin{tabular}{ll}
\hline Tax & Nature of Tax \\
\hline 3. Taxes on property & See 3.42. \\
& \\
& \\
& \\
& \\
& \\
3.2 Tax on unutilized & This tax, which came into force on \\
Land Tax Order, 1974, & June 1, 1975, is levied by a Land \\
King's Order-In-Council & $\begin{array}{l}\text { Taxation Board on land deemed } \\
\text { underdeveloped after a hearing } \\
\text { initiated by the Minister of }\end{array}$ \\
Agriculture (for agricultural land) or \\
the Minister of Local Administration \\
(for urban land). Tax may be levied \\
on all or part of a property owner's \\
land.
\end{tabular}

Exemptions and Deductions

\section{duties \\ All death and succession duties under the Death Duties Act, 1942, were abolished by the Death Duties (Repeal) Act, 1985.}

\subsection{Property transfer tax}

\subsection{Transfer duty,} Chapter 107 of the Laws, Revised Edition, 1959.
Duty is levied on the sale or longterm lease of fixed property situated in Swaziland. The person liable for payment of the tax is the party acquiring title to the property, or entering into a lease of 25 years duration (or longer), or entering into the lease of a claim for mineral rights for any period. The base for this tax is the value of the property being acquired or leased.
Exemptions include, inter alia, transfers of property by gift for public, municipal, religious, or charitable uses. Also, government purchases and purchases by public hospitals (for the sole use of the hospital) are exempt. Additionally, settlement of jointly owned property between married persons upon divorce, or on the death of one party, is exempt.
Rates

The rate of tax varies with the size, or dutiable value, of the estate. The formula for determining the rate is as follows: for every E 200 (or part thereof) in dutiable value, the tax rate rises by 0.015 percent. This is subject to a maximum tax rate of $33 \frac{1}{3}$ percent (reached at a dutiable estate value of E 445,667).

The rate of this duty is 2 percent on the first E 40,000 of transferred property value and 4 percent of any additional E 20,000 and 6 percent on the residual. 
Table 40. Swaziland: Summary of the Tax System as of December 31, 2005 (continued)

(All amounts in emalangeni)

\begin{tabular}{ll}
\hline Tax & Nature of Tax \\
\hline 3.42 Mineral rights tax. & Holders of mining rights are subject \\
Mineral Rights Tax, & to taxes with respect to properties \\
Order No. 34, 1973. & able to produce precious and \\
& nonprecious metals to which they \\
& hold rights. There are three distinct \\
& taxes that are grouped together: (a) a \\
& tax on the transfer of mining rights; \\
& (b) a ground tax on mineral rights; \\
& and (c) a capital gains tax.
\end{tabular}

4. Taxes on goods and services

\subsection{Sales tax \\ Sales Tax Act (Act. No. 12 of 1983).}

4.2 Selective excises Customs and Excise Act (Act No. 21 of 1971).

able to produce precious and nonprecious metals to which they hold rights. There are three distinct taxes that are grouped together: (a) a (b) a ground tax on mineral rights; and (c) a capital gains tax

A tax levied at the import and manufacturing levels. To be collected on certain services and all goods other than those specifically exempted. Where payable on goods imported from outside the customs union area, valuation is 110 percent of customs value plus customs duties payable. Proceeds received are directly paid to the Swazi government, and are not paid into the SACU pool.

Specific duties are payable by the importer or manufacturer of beer, tobacco, and cigarettes. Rates are

Exemptions and Deductions

\section{None.} those set by South Africa, with proceeds pooled under the Customs Union Agreement.
Many exemptions: necessities and intermediate goods for manufacturing are all mostly exempt along with certain medical supplies, temporary imports, certain personal imports, electricity, etc.

Exemptions are extended to exported goods.
Rates

(a) Transfer of mining rights is taxed at the rate of $271 / 2$ percent on the first E 20,000 of transferred value and $371 / 2$ percent above E 20,000.

(b) The tax on unexploited rights is E 10 per ha. in each of the first five years, rising to E 50 per ha. thereafter, if there has been no exploitation.

(c) The tax on gains from shares in mineral rights is $371 / 2$ percent of that gain.

14 percent on imported and locally manufactured or produced goods.

25 percent on all kinds of alcoholic beverages (imported or locally brewed), except traditional beer.

14 percent on traditional beer. 14 percent on accommodation let $\&$ food supplied by hotels or restaurants.

20 percent on locally manufactured tobacco products.

25 percent on imported tobacco products.

Rates of excise duty are mostly specific. Examples of these rates include the following:

(1) malt beer $340 \mathrm{ml}$ E 43.57;

(2) Sorghum beer 1 liter E 82;

(3) Spirits (average) $750 \mathrm{ml}$ E 1,184.00;

(4) Sparkling wine 1 liter E 227.60;

(5) Fortified wine 1 liter E 182.50;

(6) Unfortified wine 1 liter E 80.70;

(7) Cigarettes 20 E 350.80;

(8) Pipe tobacco 25 grams E 131.30. 
Table 40. Swaziland: Summary of the Tax System as of December 31, 2005 (continued)

(All amounts in emalangeni)

\begin{tabular}{|c|c|c|c|}
\hline Tax & Nature of Tax & Exemptions and Deductions & Rates \\
\hline $\begin{array}{l}4.3 \text { Business and } \\
\text { professional licenses } \\
\text { Trading Licence Act, } \\
1975 .\end{array}$ & $\begin{array}{l}\text { Annual license fees are charged for } \\
\text { betting shops, companies with a } \\
\text { place of business in Swaziland, } \\
\text { persons or companies trading in } \\
\text { Swaziland, and establishments } \\
\text { licensed to sell or serve liquor. }\end{array}$ & None. & $\begin{array}{l}\text { Annual fees areas follows: } \\
\text { - } \quad \text { Betting licenses are } \\
\text { charged on the basis of } \\
\text { annual turnover. } \\
\text { - Company license fees } \\
\text { vary from E } 20 \text { per annum } \\
\text { for companies with a } \\
\text { share capital of less than } \\
\text { E } 10,000 \text { to E } 200 \text { per } \\
\text { annum for companies } \\
\text { with a share capital above } \\
\text { E 50,000. } \\
\text { Trading licenses are } \\
\text { subject to a wide variety } \\
\text { of rates, depending on the } \\
\text { trade carried out, but are } \\
\text { generally between E } 50 \\
\text { and E } 5000 \text { per annum } \\
\text { (amended schedule, April } \\
\text { 2005). } \\
\text { *The Trading Licences( } \\
\text { Amendment of Schedule } \\
\text { B) Regulations } 2003 \\
\text { cost between rural and } \\
\text { nonrural areas, with the } \\
\text { type of establishment, and } \\
\text { the type of beverage sold; } \\
\text { they are between E } 25 \text { and } \\
\text { E } 750 \text { per annum. }\end{array}$ \\
\hline
\end{tabular}


Table 40. Swaziland: Summary of the Tax System as of December 31, 2005 (continued)

(All amounts in emalangeni)

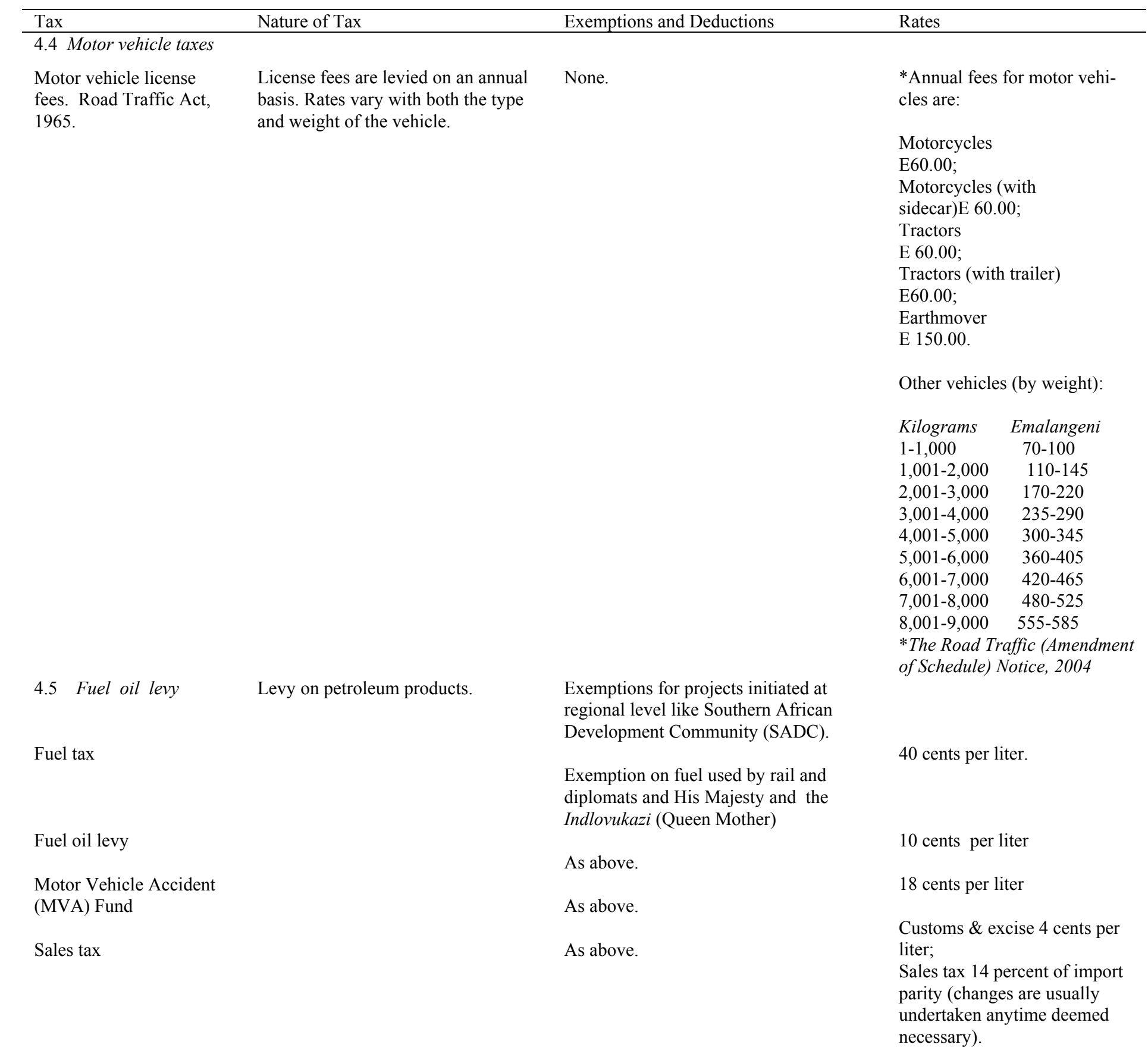


Table 40. Swaziland: Summary of the Tax System as of December 31, 2005 (continued) (All amounts in emalangeni)

\begin{tabular}{ll}
\hline Tax & Nature of Tax \\
\hline $\begin{array}{l}\text { 5. Taxes on international } \\
\text { trade and transactions }\end{array}$ & \\
$\begin{array}{l}\text { 5.1 Duties on imports } \\
\text { Customs Union }\end{array}$ & $\begin{array}{l}\text { A common taxation system is levied } \\
\text { on imports in conjunction with }\end{array}$ \\
$\begin{array}{l}\text { Agreement, 1969 Legal } \\
\text { Botice (No. 71 of 1969) }\end{array}$ & $\begin{array}{l}\text { South Africa. Import duties are } \\
\text { and Customs, Fiscal, } \\
\text { Excise, and Sales Duties }\end{array}$ \\
$\begin{array}{l}\text { levied at the point of entry into the } \\
\text { common customs area and thereafter } \\
\text { 1971). }\end{array}$ & $\begin{array}{l}\text { the imports are free to move within } \\
\text { the entire area. The importer is }\end{array}$ \\
& $\begin{array}{l}\text { legally liable for the payment of } \\
\text { these duties, the proceeds of which } \\
\text { are pooled and divided among the } \\
\text { countries according to a formula. }\end{array}$ \\
& $\begin{array}{l}\text { Duty rates are set by South Africa on } \\
\text { the basis of the six-digit Harmonized } \\
\text { Commodity Description and Coding }\end{array}$ \\
& $\begin{array}{l}\text { System. The rate structure includes } \\
\text { general and most-favored-nation } \\
\text { clauses. Most duties are ad valorem }\end{array}$ \\
& with specific duties on some items.
\end{tabular}

\subsection{Taxes on exports}

\subsection{Sugar levy. Sugar} Export Levy Act No. 4 of 1997.

Exemptions and Deductions

Rebates, remissions, and refunds of import duties are allowed in some cases (mostly for raw materials and semimanufactures). As with duty rates, such rebates must conform to South African rebates.
The sugar export levy is a tax on all None. sugar exported from Swaziland to the EU. The levy is collected from the millers and growers by the Swaziland Sugar Association (SSA), which then remits it on a quarterly basis to the government.

\subsection{Cattle export} slaughter tax. Details not available.

\section{Other taxes}

\subsection{Stamp taxes} Chapter 100 of the Laws, 1959. The Stamp Duties Act, 1970 (Act No. 37) and 1974 (Act No. 13), as amended by the Finance Act of 1985.
There are 45 ad valorem rates, ranging from 0 to over 70 percent.
The levy is on the net ex-mill protocol sales to the European Union to be applied two years in arrears. Net ex-mill export protocol sales proceeds are the Swazi currency equivalent of the gross amount received by the association in respect of all sales of sugar exported, less expenses as prescribed in the act and is payable on a quarterly basis.

No longer enforced, but not yet repealed.

Government and specified public $\begin{array}{ll}\text { These taxes, which are mostly ad } & \text { Governmen } \\ \text { valorem with some specific taxes, are } & \text { enterprises. }\end{array}$ payable on a wide range of legal documents (affidavits, bills of exchange, checks, bonds, contract notes, receipts, property transfers, etc.).
Stamp duties vary considerably. For example, checks carry a 6 percent stamp duty, receipts for payments of $\mathrm{E} 2$ or more carry an E 0.10 duty, customs bills of entry an $\mathrm{E}$ 0.40 duty, and affidavits, agreements, and contracts an E 1 stamp duty. 
Table 40. Swaziland: Summary of the Tax System as of December 31, 2005 (continued)

(All amounts in emalangeni)

\begin{tabular}{lll}
\hline Tax & Nature of Tax & Exemptions and Deductions \\
\hline $\begin{array}{l}\text { 6.2 Miscellaneous } \\
\text { licenses }\end{array}$ & These are charged for dog licenses. & $\begin{array}{l}\text { None, but the law is not enforced; } \\
\text { however, it has not been repealed. }\end{array}$ \\
$\begin{array}{l}\text { Act, 1953, as amended by } \\
\text { the Finance Act, 1985. }\end{array}$ & & $\begin{array}{l}\text { Dog license tax is E 1 per } \\
\text { annum in rural areas and E 3 } \\
\text { per annum in urban areas. }\end{array}$ \\
$\begin{array}{l}\text { B. Local taxes } \\
\text { Property tax. Legal refer- } \\
\text { ence not available. }\end{array}$ & $\begin{array}{l}\text { Taxation in the form of rates is } \\
\text { collected in the two principal towns } \\
\text { (Mbabane and Manzini). Land and }\end{array}$ & $\begin{array}{l}\text { Exemptions include government-owned } \\
\text { property. }\end{array}$ \\
& $\begin{array}{l}\text { improvements are taxed at different } \\
\text { rates with quinquennial valuation } \\
\text { assessments. If changes in tax rates } \\
\text { are desired by the town councils, } \\
\text { approval is required by the central } \\
\text { government before such changes can } \\
\text { be enforced. }\end{array}$ & \\
&
\end{tabular}


Table 40. Swaziland: Summary of the Tax System as of December 31, 2005 (concluded)

(All amounts in emalangeni)

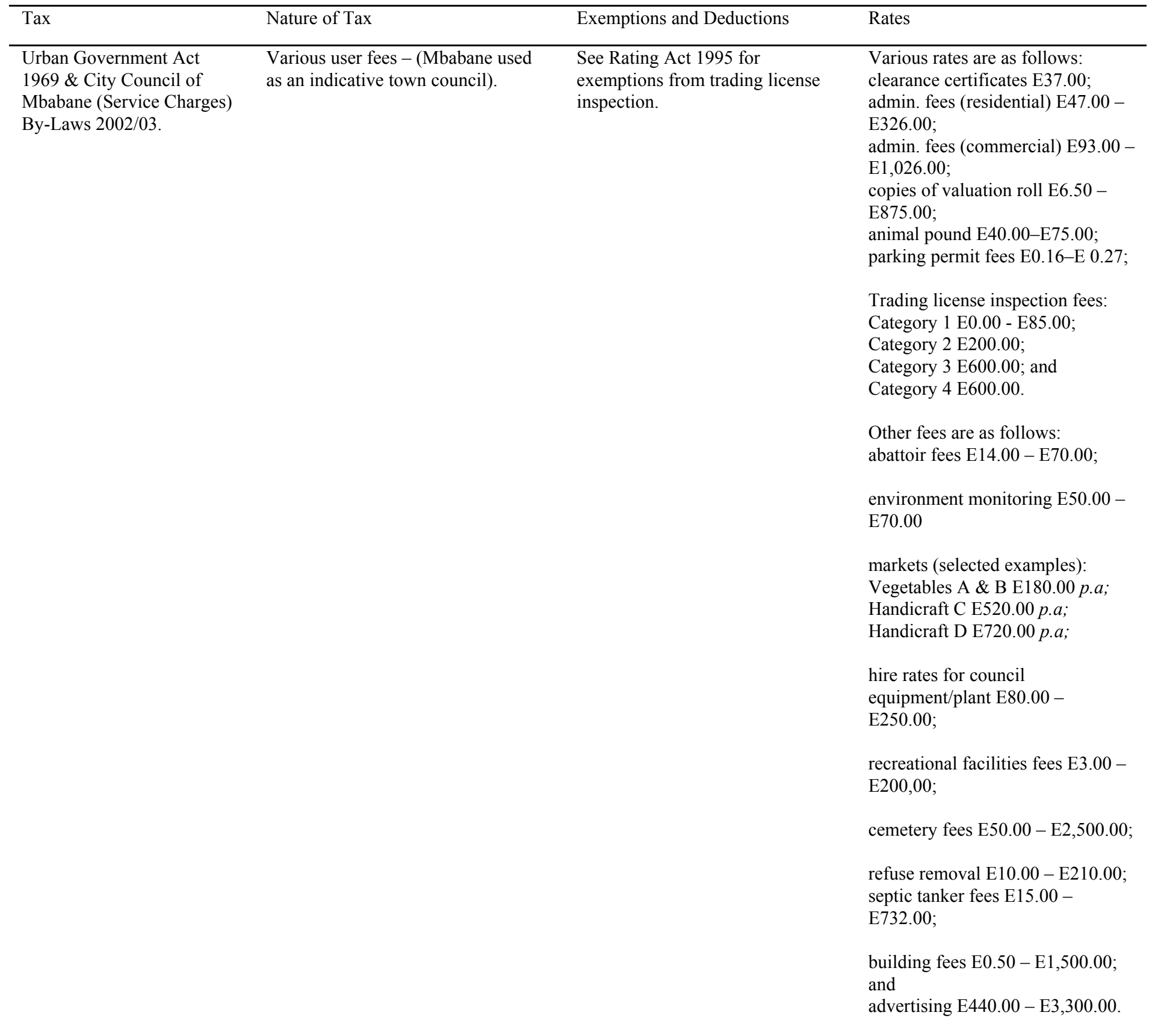

Sources: United Kingdom Board of Internal Revenue, Income Taxes Outside the United Kingdom, Vol. 7 (1972); International Bureau of Fiscal Documentation, Tax System; International Monetary Fund, Surveys of African Economies, Vol. 5; and Swaziland, Government Information Services, A Handbook to the Kingdom of Swaziland, various years, updated by the Swazi authorities. 